\title{
Antibiotic cement coating in orthopedic surgery: a systematic review of reported clinical techniques
}

\author{
Abdullah Ismat ${ }^{1}$, Nike Walter', Susanne Baertl', Joerg Mika², Siegmund Lang ${ }^{1}$, Maximilian Kerschbaum', \\ Volker Alt ${ }^{1}$ and Markus Rupp ${ }^{1 *}$ (D)
}

\begin{abstract}
Background: Antibiotic-containing cement and bone graft substitute-coated orthopedic implants provide the advantages of simultaneous local antibiotic delivery and internal stable fixation, aiding in both infection eradication and osseous healing. Standardized protocols pertaining to implant coating techniques in various clinical and particularly intraoperative settings are scarce, and available literature is limited. This systematic review aims to provide a summary of the available current literature reporting on custom-made coating techniques of orthopedic implants, indications, outcomes, and associated complications in clinical use.
\end{abstract}

Methods: A systematic search of the literature in PubMed, Medline, Embase, and Cochrane Library databases was performed in accordance with PRISMA guidelines. Articles reporting specifically on custom-made coating techniques of orthopedic implants in a clinical setting were eligible.

Results: A total of 41 articles with a cumulative total number of 607 cases were included. Indications for treatment mostly involved intramedullary infections after previous plate osteosynthesis or nailing. A variety of implants ranging from intramedullary nails, plates, wires, and rods served as metal cores for coating. Polymethylmethacrylate (PMMA) bone cement was most commonly used, with vancomycin as the most frequently added antibiotic additive. Chest tubes and silicone tubes were most often used to mold. Common complications are cement debonding and breakage of the metallic implant.

Conclusion: Adequate coating techniques can reduce the burden of treatment and be associated with favorable outcomes. Lack of general consensus and heterogeneity in the reported literature indicate that the perfect all-in-one implant coating method is yet to be found. Further efforts to improve implant coating techniques are warranted.

Level of evidence: III.

Keywords: Bone cement coating, Implant coating, Osteosynthesis, Osteomyelitis

\section{Introduction}

The use of bone cement in orthopedics has become integral to many operative procedures. Its first practical use was reported around 60 years ago [1], where it was

\footnotetext{
*Correspondence: Markus.rupp@ukr.de

1 Department of Trauma Surgery, University Medical Center Regensburg,

Franz-Josef-Strauß-Allee 11, 93053 Regensburg, Germany

Full list of author information is available at the end of the article
}

primarily used in joint replacement surgeries. Over the years, the spectrum of applications of bone cement has been growing. Developments in research and quality of bone cement as well as its delivery methods and systems have additionally contributed to its being employed as a local drug delivery agent.

Indications for cement coating include not only enhanced fixation of implants but also infection prophylaxis and treatment through local application of additive 
therapeutic agents including antibiotics [2]. Buchholz et al. mentioned the use of antibiotics as additives in bone cement back in 1970 [3].

Contrary to readily available antibiotic-containing products including beads, initially developed by Klemm et al. [4] in 1979, which only contain gentamicin [5], a wider range of antibiotic agents can be added to polymethylmethacrylate (PMMA) cement in accordance to the susceptibility of the causative organisms [6]. Aside from joint-replacing prosthesis, cement coating has been reported in other internal fixation methods and orthopedic implants including plates, wires, and rods [7-9].

Nevertheless, standardized clinical protocols and reports pertaining to cement coating techniques for different orthopedic implants in various operative settings are lacking in literature.

The aim of this review is to outline reported custommade methods of cement coating techniques, indications, outcome, and complications associated with their application. The resulting insights into the particularities relevant to the various techniques should help to improve treatment delivery methods and outcomes in daily clinical practice.

\section{Methods}

This systematic review was registered in the PROSPERO international prospective register of systematic reviews (registration no. CRD42021236015). The Preferred Reporting Items for Systematic Reviews and Meta-Analyses (PRISMA) 2009 checklist was adhered to (Fig. 1).

A search of literature reporting on coating techniques for orthopedic implants in different clinical settings was performed using PubMed, Medline, Embase, and Cochrane Library. Published articles ranging from case reports, case series, clinical trials, and review articles were initially analyzed. Articles published in English and German were reviewed. The search terms: "bone cement coating," "bone implant coating," "implant cement coating," "antibiotic cement nail," and "antibiotic cement rod" were used in the different literature databases to identify

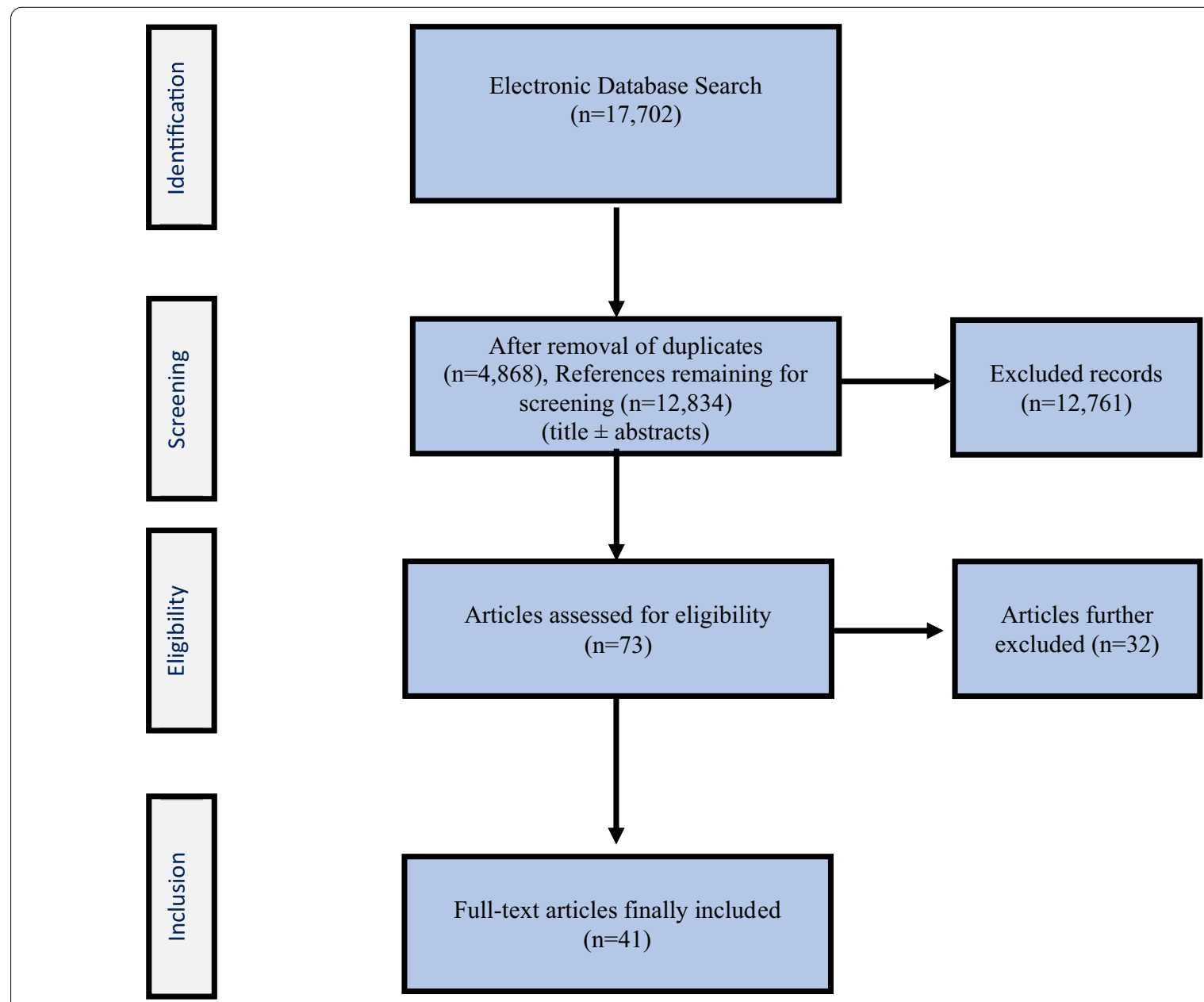

Fig. 1 PRISMA flow diagram presenting the methodological approach for identification, screening, eligibility, and final inclusion of relevant articles 
relevant articles. Articles had to include a clear outline of the custom-made cement or bone substitute coating technique applied to the orthopedic device being implanted in a clinical setting. The articles' titles and abstracts were initially screened for relevance.

Cohort size, type of orthopedic implants coated, indications for cement coating, type of cement coating, used additives, and technical details of the cement coating techniques were noted. A table serving as a summary of each article reporting on coating techniques was then constructed to serve as a practical guide reflecting currently available evidence (Table 1 ). Differences in the mentioned techniques of cement coating were compared and discussed thoroughly. A total of 9118 and 8584 references were initially identifiable using the different search terms in the PubMed and Embase databases, respectively. In the Cochrane Library, the search results provided the same 2 articles under all search terms, which were not eligible for inclusion and directly excluded. Thus, a total of 17,702 (9118 from PubMed +8584 from Embase) were identified. From this total of 17,702 references, 4868 duplicates were identified and removed, resulting in 12,834 remaining references.

The titles and abstracts of each of the remaining references were screened for eligibility. Reviews, experimental studies, nonclinical articles, conference papers, and clinical articles reporting on precoated as well as commercially prefabricated coated orthopedic implants and ones pertaining to dental implants were excluded $(n=12,761)$. Articles reporting on use of cement or bone graft substitute to coat orthopedic implants in a clinical setting were included. This left a total of 73 articles, among which 58 full texts were available. From these 58 available full-text articles, articles reporting on and specifically outlining custom-made coating techniques as part of their surgical treatment method in an intraoperative setting were included. This resulted in the final inclusion of 41 fulltext articles for appraisal (Fig. 1).

\section{Indications}

The most common reported indications for use of cement-coated implants were intramedullary (IM) infections of long bones after previous osteosynthesis or IM nailing and infected nonunions (Table 2).

Antibiotic-impregnated cement-coated implants were also used to treat chronic osteomyelitis with and without bone defects after debridement. Chronic infections after total knee replacements and shoulder prosthesis have also been treated with coated implants. Additionally, this treatment method was used to treat chronic osteomyelitis and infected Charcot ankles.

In the acute setting, indications for cement-coated implants included infected open fractures, early infections following fracture plate osteosynthesis, and treatment of polytrauma patients requiring external fixation as part of damage control orthopedics and for medullary infection prevention.

\section{Coating techniques}

Various orthopedic implants have been used as metal cores in the coating process (Table 3). Early reports, published almost 20 years ago, utilized intramedullary guidewires of $3 \mathrm{~mm}$ thickness [10]. This allowed for some stability across fracture sites and infected bones but was limited and inadequate for weight-bearing and definitive bone healing. Other metal cores, also of limited stability, used included K-wires [11-14], Ilizarov wires [12, 15], steel wires [16], ball-tipped guide wires [15], and Steinmann pins $[17,18]$. To provide more stable constructs and allow for simultaneous weight-bearing, other groups reported on the use of IM nails [19-25] of long bones for coating. This mainly included antegrade and retrograde femoral IM nails as well as tibial IM nails. Furthermore, clinically beneficial constructs were also created with use of knee and ankle arthrodesis implants $[19,26]$, and some authors even reported coating of inverse shoulder prosthesis and plates used for revisions of septic loosening and for fracture fixation [8], respectively. To provide more protection across the fusion site and aid in early weight-bearing, some authors reported using antibioticcontaining coated IM nails in combination with simultaneous external ring fixation in cases of unstable infected neuropathic ankles in obese patients [27].

\section{Type of bone cement/bone graft substitute and quantity of antibiotic additives used in different studies}

PMMA cement was the bone cement most commonly used $(n=34)$ to coat orthopedic implants in a custommade fashion, with Palacos $(n=7)$ and Simplex $(n=7)$ formulations being the most frequently used and mixed with antibiotic additives (Table 4). Some studies $(n=6)$ also utilized premixed bone cement formulations containing most commonly premixed gentamicin $(n=6)$ or premixed tobramycin $(n=1)$. More recently, certain groups $[8,26]$ reported on the application of Cerament (Bonesupport AB, Lund, Sweden) as a coating, a bone graft substitute consisting of calcium sulfate and calcium hydroxyapatite, premixed with either gentamicin or vancomycin.

Antibiotic additives which were mixed with the bone cement to form the coating mantle of orthopedic implants most commonly involved the use of vancomycin, either alone $(n=7)$ or in combination with other antibiotic agents $(n=24)$. Vancomycin was most frequently mixed with tobramycin $(n=16)$ followed by gentamicin $(n=6)$, teicoplanin $(n=1)$, and cefuroxime 


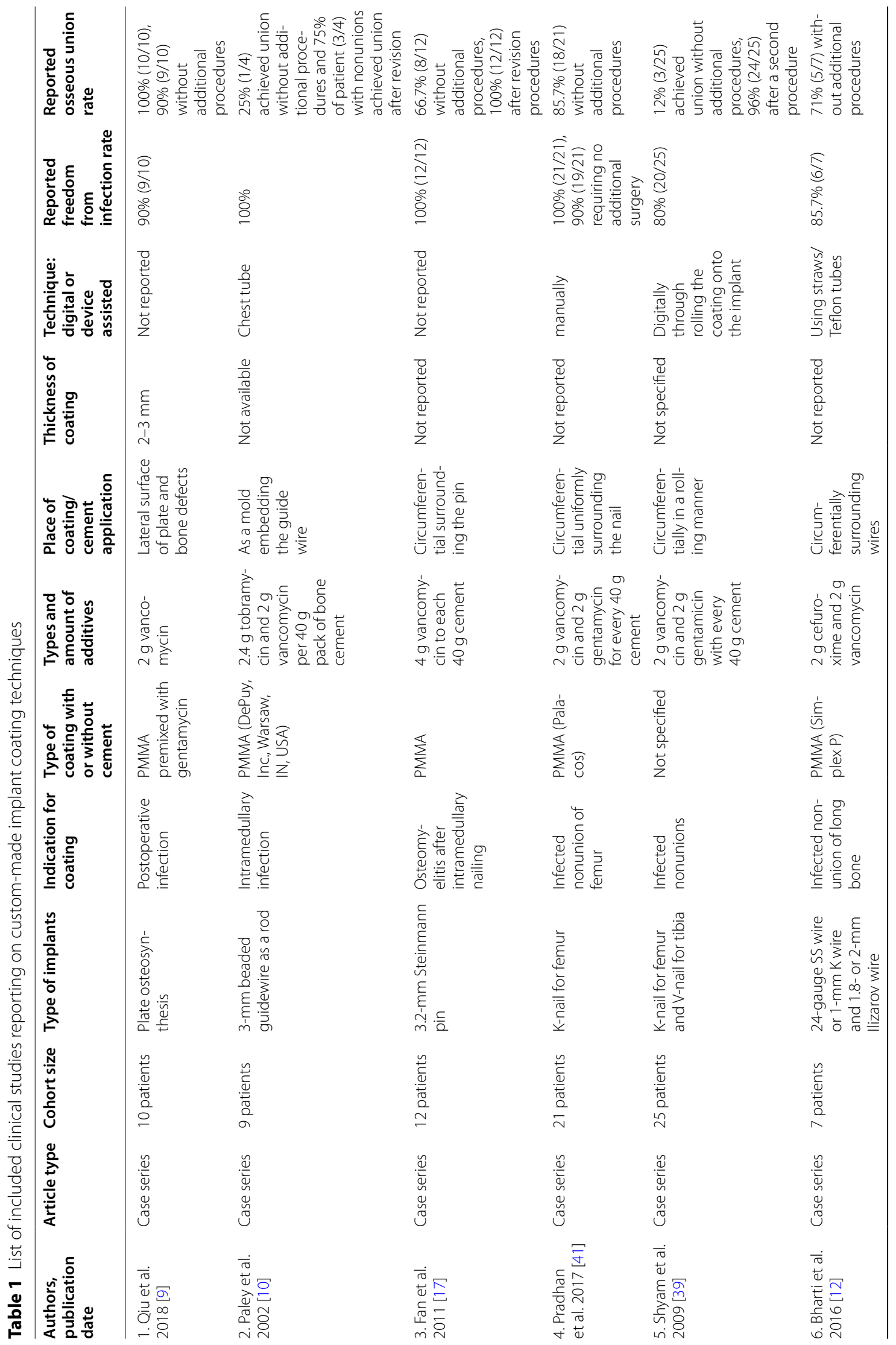



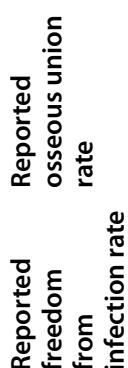

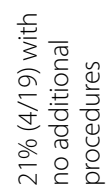

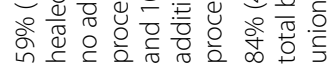

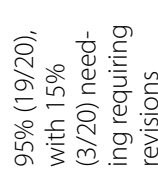

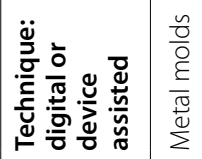

¿

峞
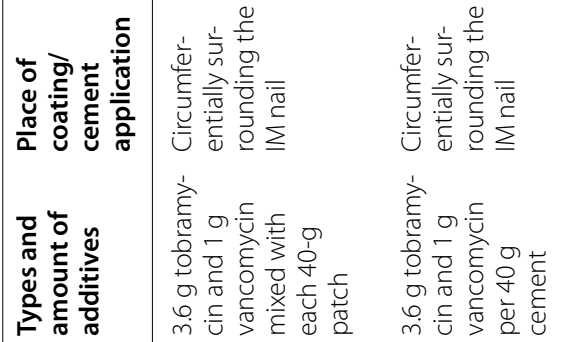

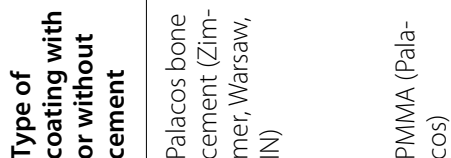

흐

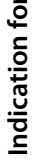

흘

\section{(2)}

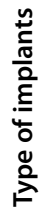

$\frac{\sqrt{2}}{\mathfrak{n}}$

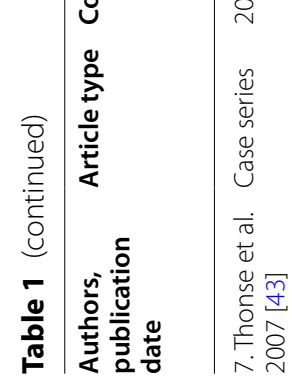

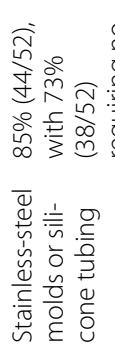

$\stackrel{\varepsilon}{\varepsilon}$

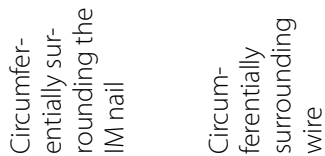

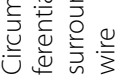

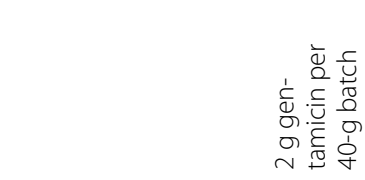

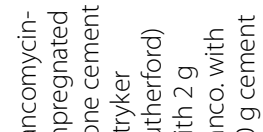

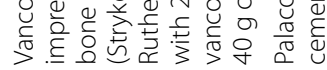

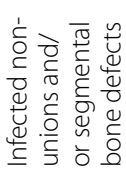

离

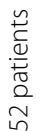

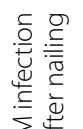

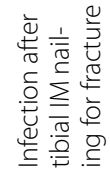

$\sum_{\substack{\frac{d}{0} \\ \frac{0}{0}}}^{\frac{0}{3}}$

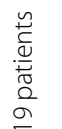

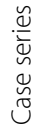

宁

$\stackrel{\sim}{\sim} \sigma$

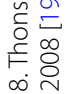

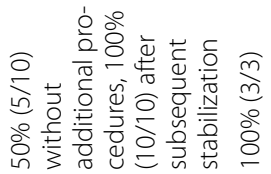

$\begin{array}{ll}\stackrel{0}{o} & \widehat{m} \\ \frac{m}{0} & \stackrel{0}{0}\end{array}$

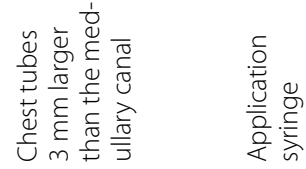

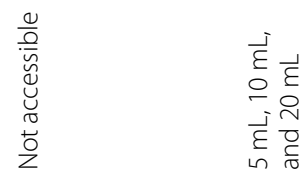

อ

莫苋

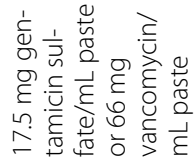

जे>

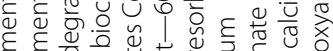

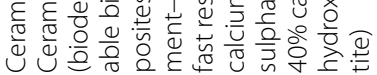

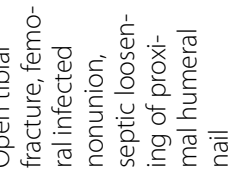

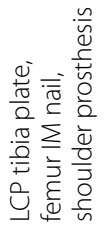

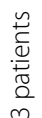

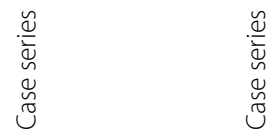

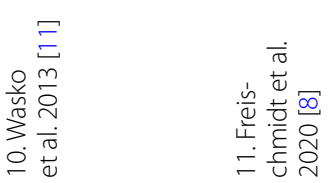




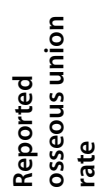

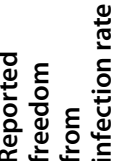

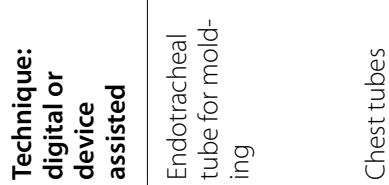

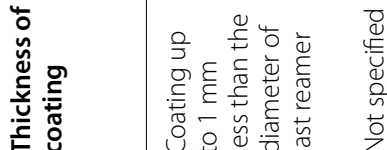
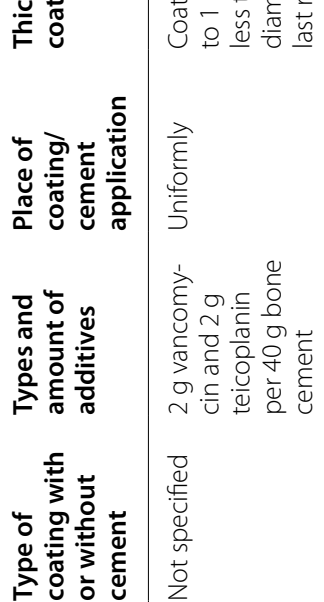

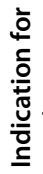

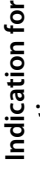

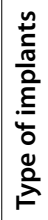

$\frac{N}{N}$
$\frac{N}{4}$
$\frac{1}{0}$
$\frac{0}{0}$

㝏

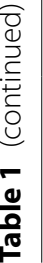

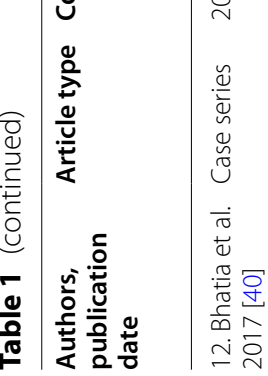

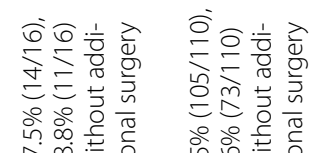

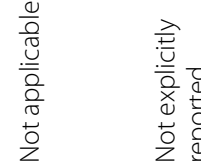

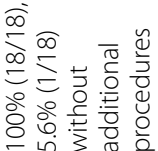

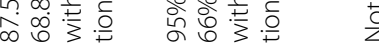

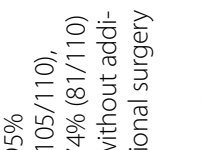

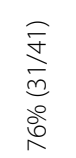

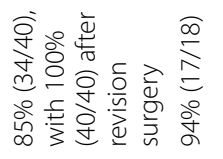

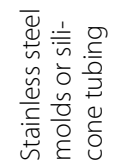

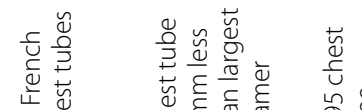

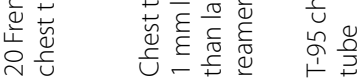

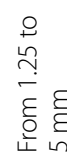

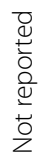

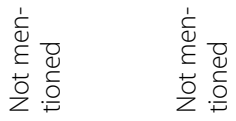

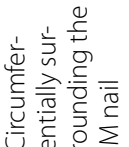

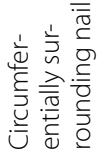

离

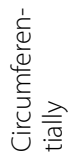

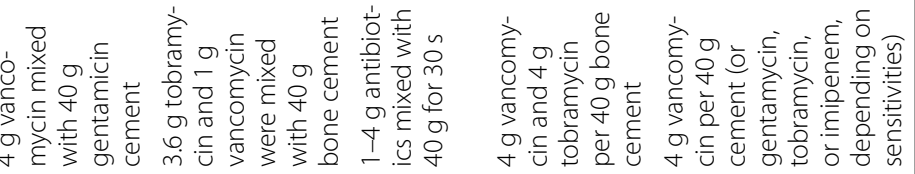

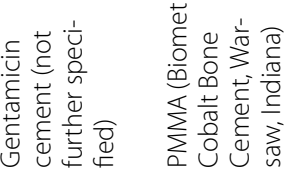

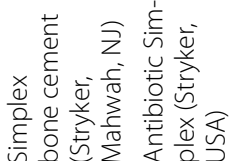

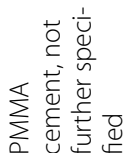

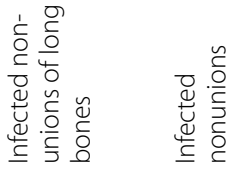

垩离

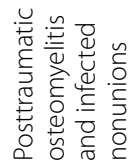

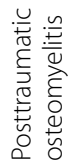

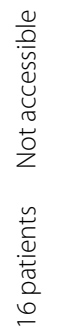

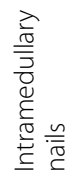

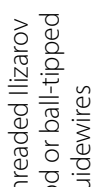

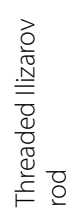

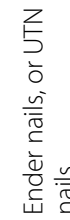

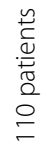

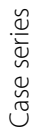

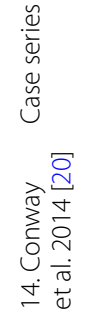

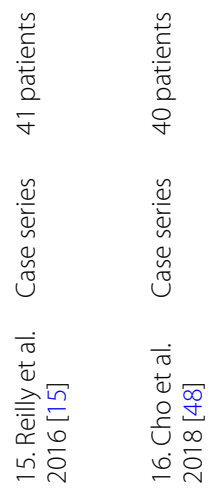

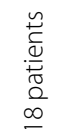

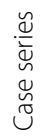

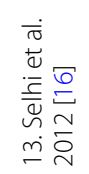

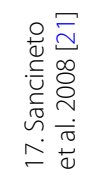




\begin{tabular}{|c|c|c|c|c|c|}
\hline 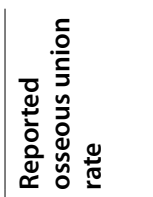 & $\begin{array}{l}\frac{0}{0} \\
\frac{0}{\overline{0}} \\
\frac{0}{0} \\
\frac{0}{0} \\
\frac{0}{2}\end{array}$ & $\begin{array}{l}\frac{0}{0} \\
\frac{0}{0} \\
\frac{0}{0} \\
\frac{0}{0} \\
\frac{0}{0} \\
\frac{0}{2}\end{array}$ & $\begin{array}{l}\frac{0}{0} \\
\frac{0}{0} \\
\frac{0}{0} \\
\frac{0}{0} \\
\frac{0}{0} \\
\frac{0}{2}\end{array}$ & $\begin{array}{l}\frac{0}{0} \\
\frac{0}{0} \\
\frac{0}{0} \\
\frac{0}{0} \\
\frac{0}{0} \\
\frac{0}{2}\end{array}$ & 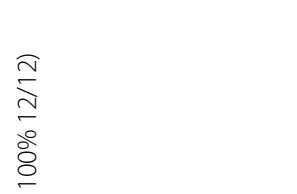 \\
\hline 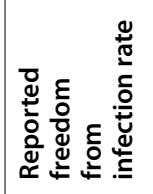 & $\begin{array}{l}\frac{0}{0} \\
\frac{0}{0} \\
\frac{0}{0} \\
\frac{0}{0} \\
\frac{0}{2} \\
\frac{0}{2}\end{array}$ & $\begin{array}{l}\frac{8}{8} \\
\frac{8}{9} \\
\stackrel{8}{\circ}\end{array}$ & $\begin{array}{l}\frac{0}{0} \\
\frac{0}{0} \\
\frac{0}{0} \\
\frac{0}{0} \\
\frac{0}{2} \\
\frac{0}{2}\end{array}$ & $\begin{array}{l}\frac{0}{0} \\
\frac{0}{\overline{0}} \\
\frac{0}{0} \\
\frac{0}{0} \\
\frac{0}{2}\end{array}$ & 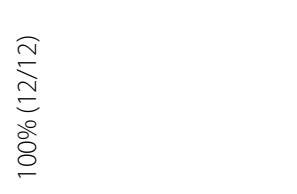 \\
\hline 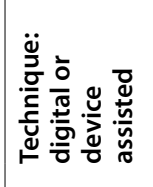 & 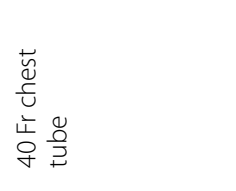 & 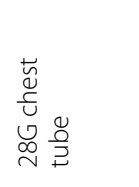 & 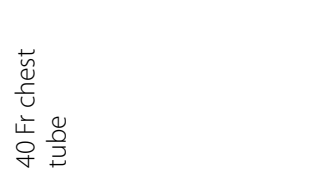 & 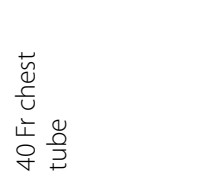 & 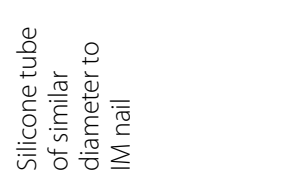 \\
\hline 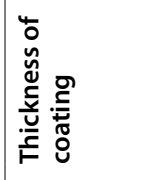 & 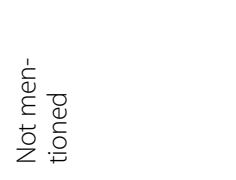 & 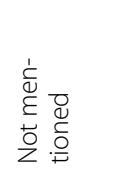 & 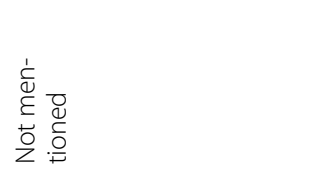 & 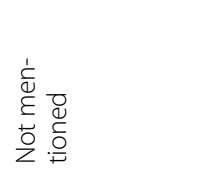 & 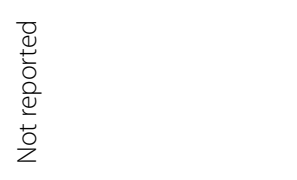 \\
\hline 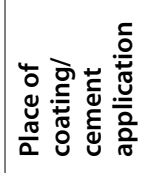 & 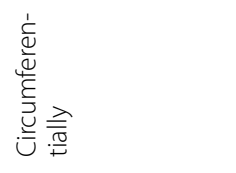 & 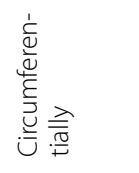 & 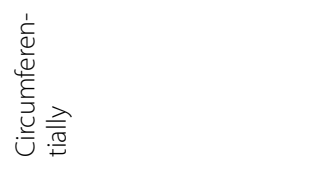 & 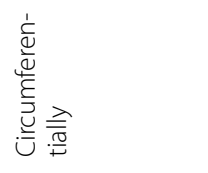 & 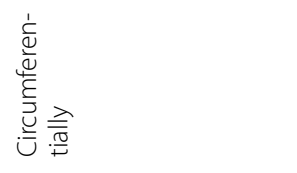 \\
\hline 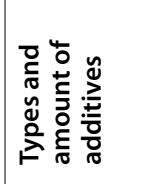 & 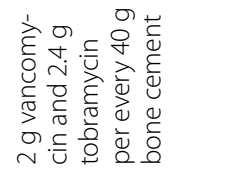 & 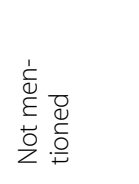 & 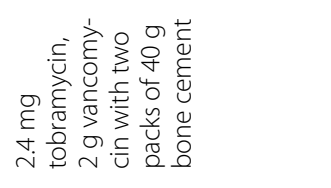 & 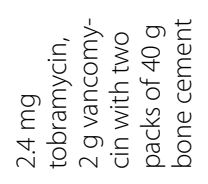 & 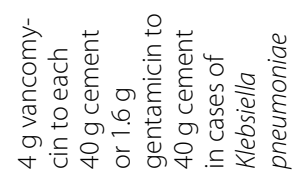 \\
\hline 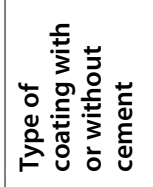 & 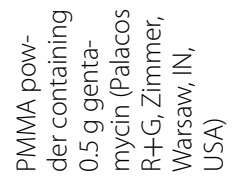 & 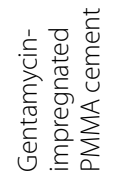 & 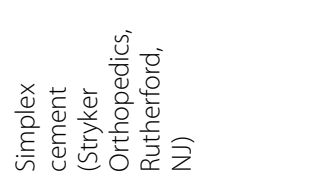 & 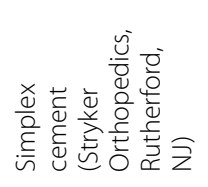 & 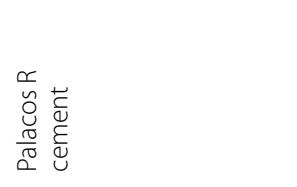 \\
\hline 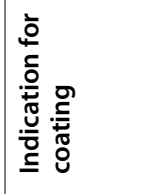 & 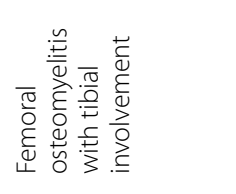 & 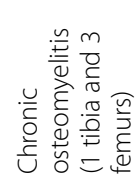 & 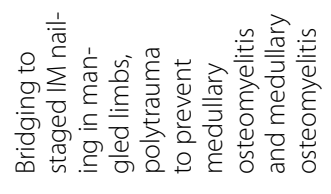 & 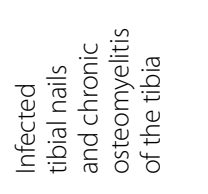 & 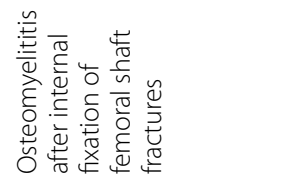 \\
\hline 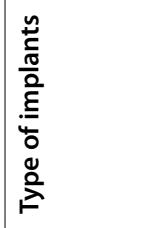 & 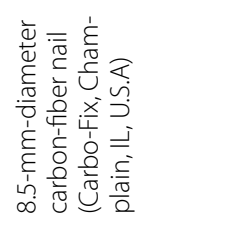 & 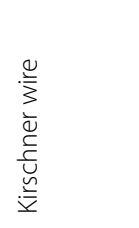 & 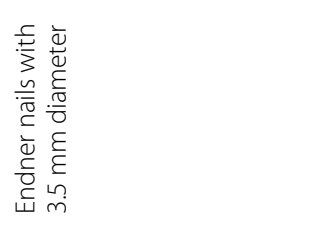 & 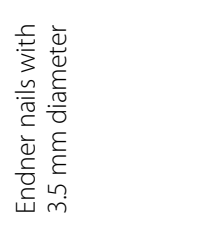 & 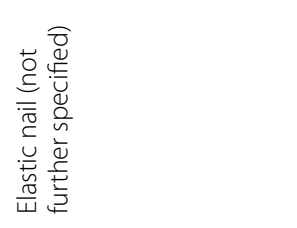 \\
\hline 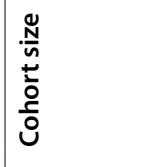 & 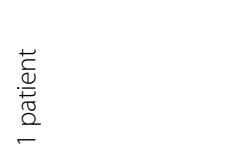 & 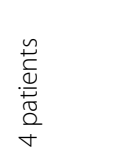 & 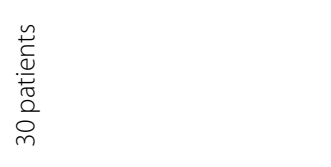 & 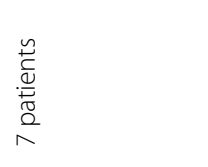 & 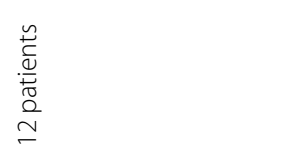 \\
\hline 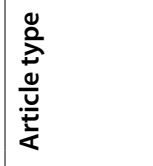 & 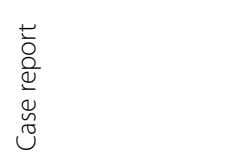 & 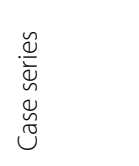 & 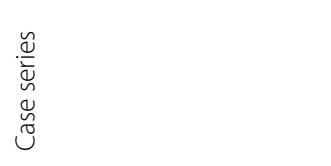 & 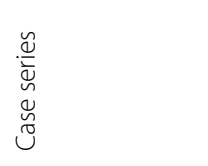 & 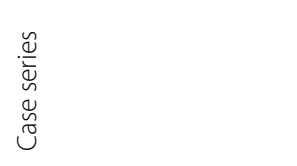 \\
\hline 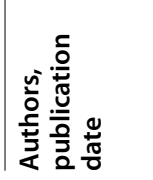 & 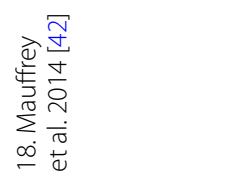 & 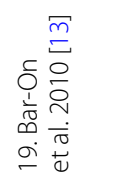 & 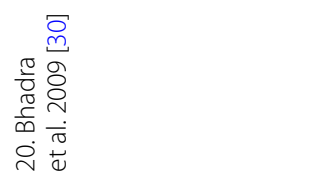 & 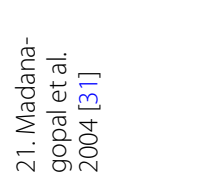 & 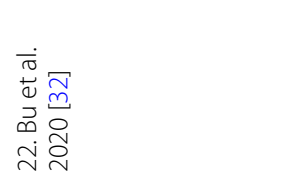 \\
\hline
\end{tabular}




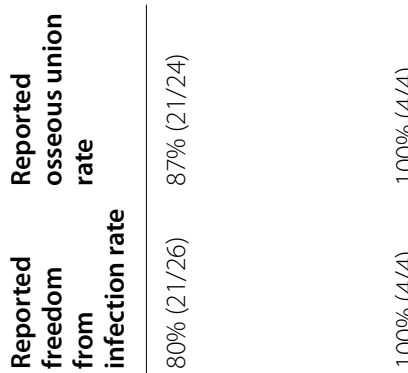

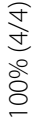

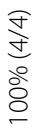

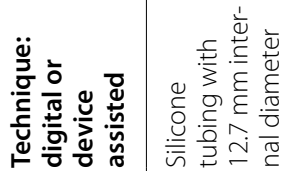

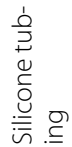

范

产

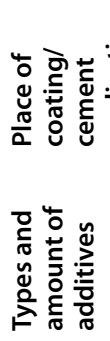

产

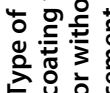

要

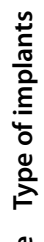

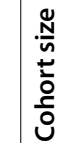

总言

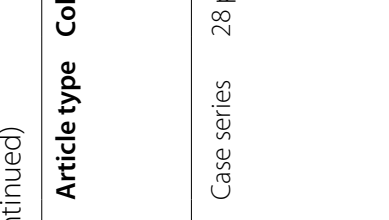

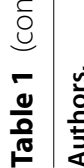

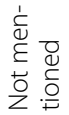

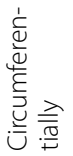

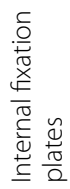

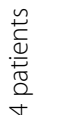

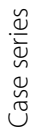

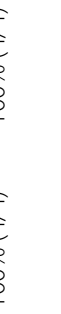

$\frac{0}{d}$
$\frac{+}{0}$
$\frac{0}{2}$
$\frac{1}{2}$
$\frac{0}{2}$

$\equiv$
$\equiv$
$\grave{\varrho}$

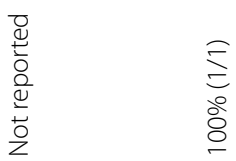

竞

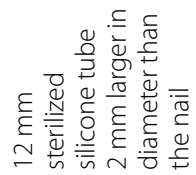

$\stackrel{\xi}{\sim}$

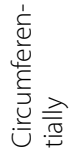

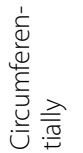

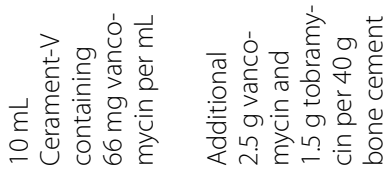

$\frac{7}{\frac{1}{2}}$

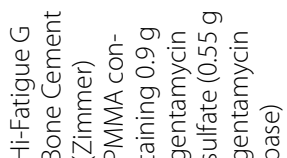

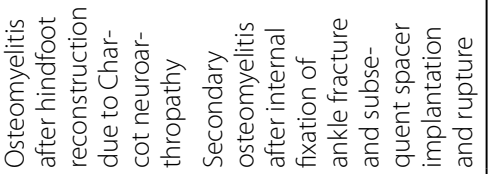

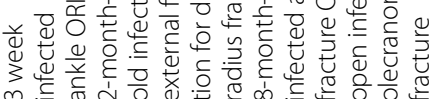

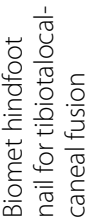

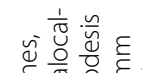

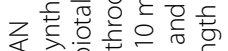

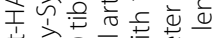

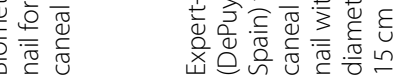

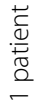

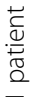

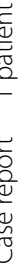

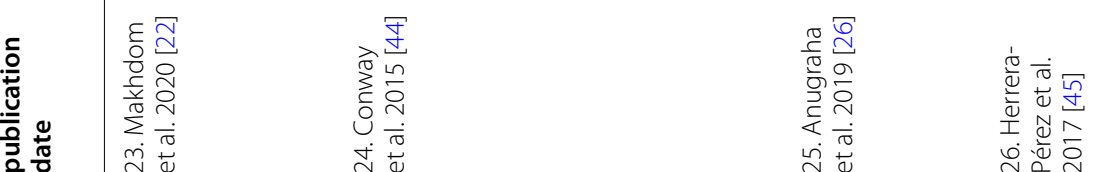




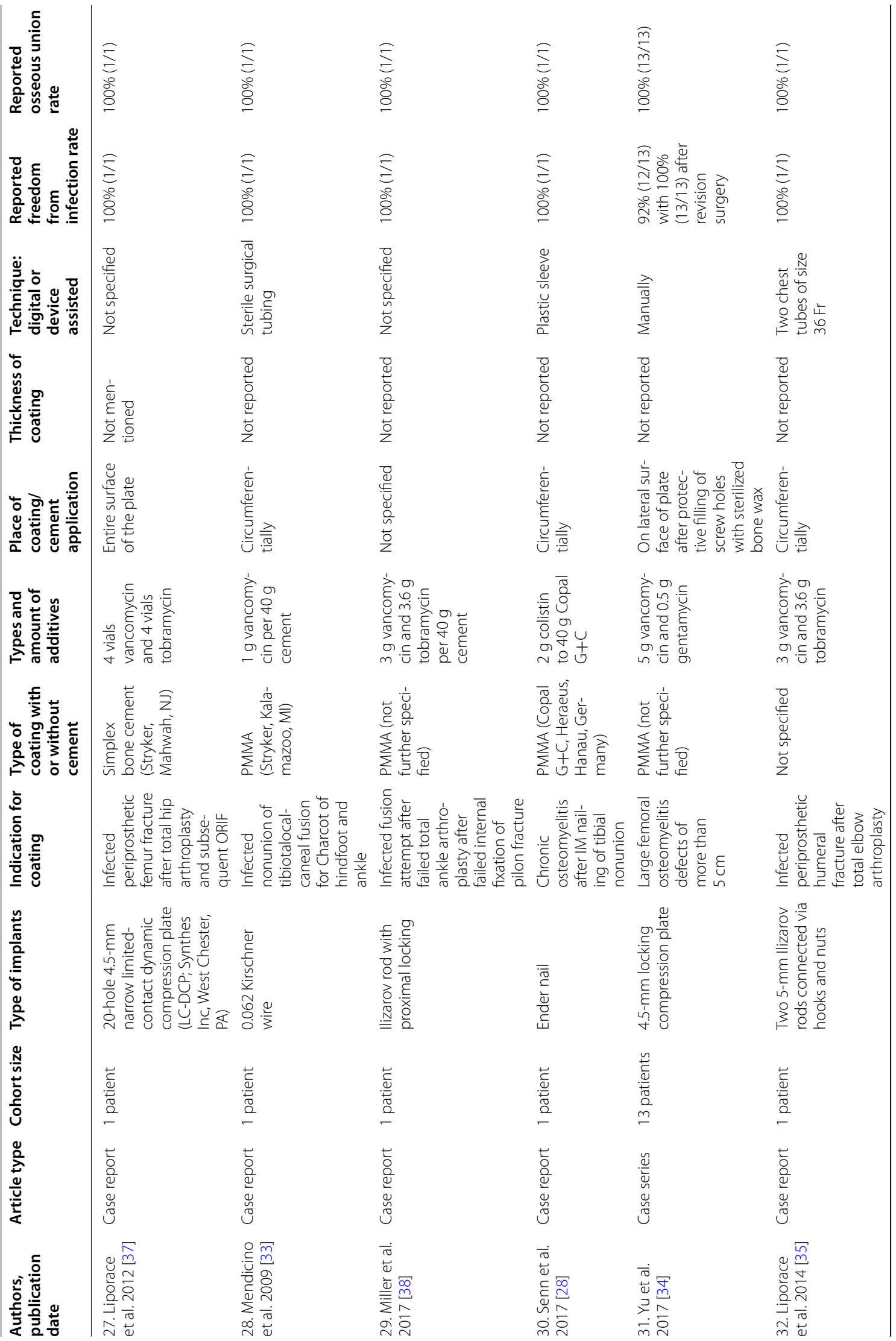



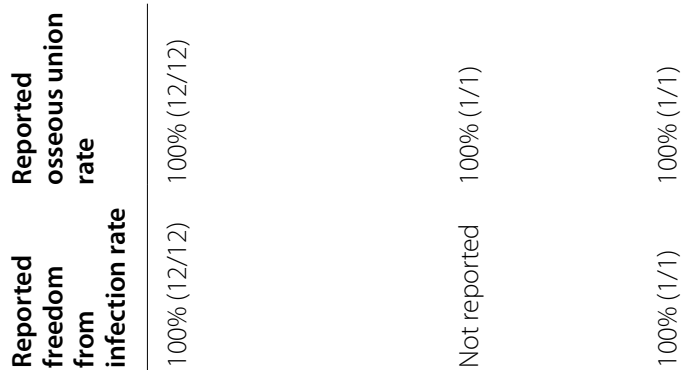

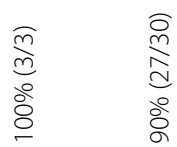

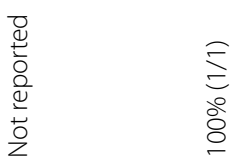

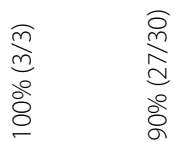

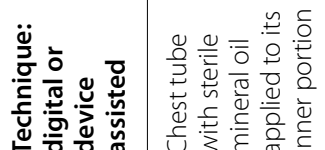

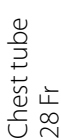

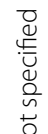

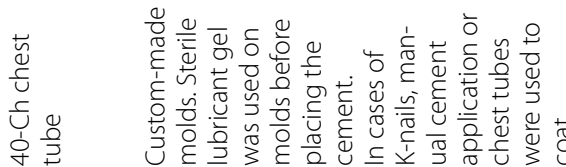
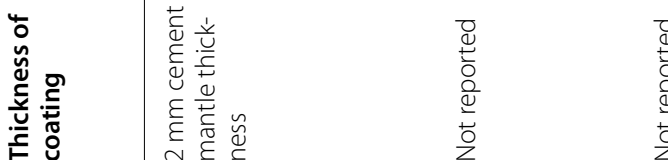

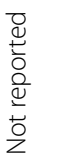
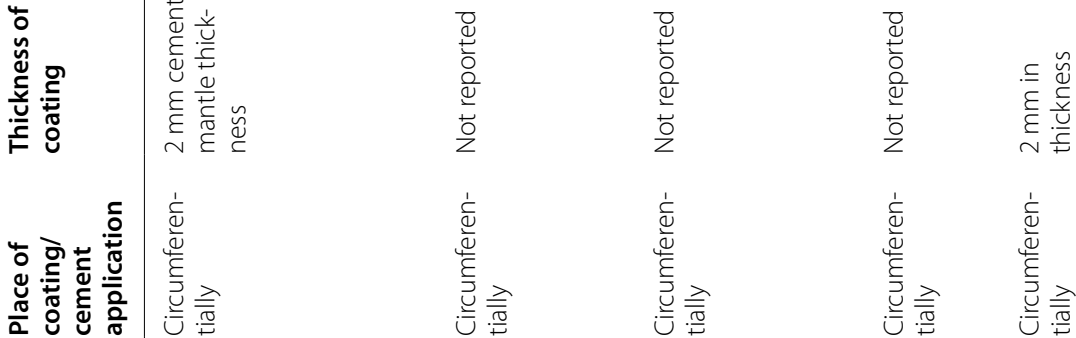

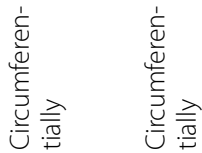
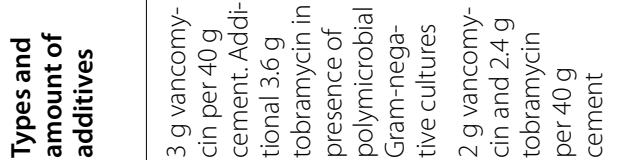

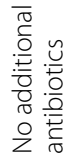

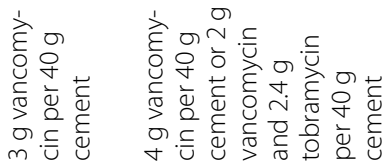

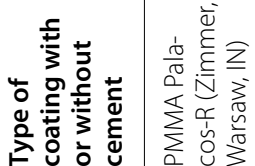

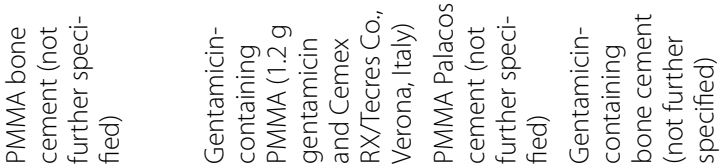

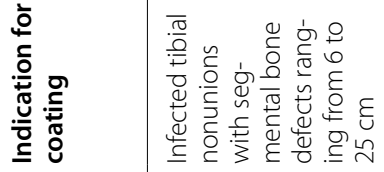

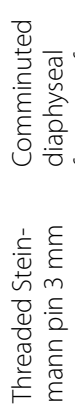

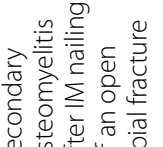

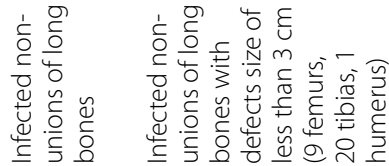

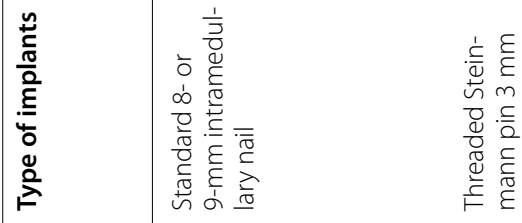

六
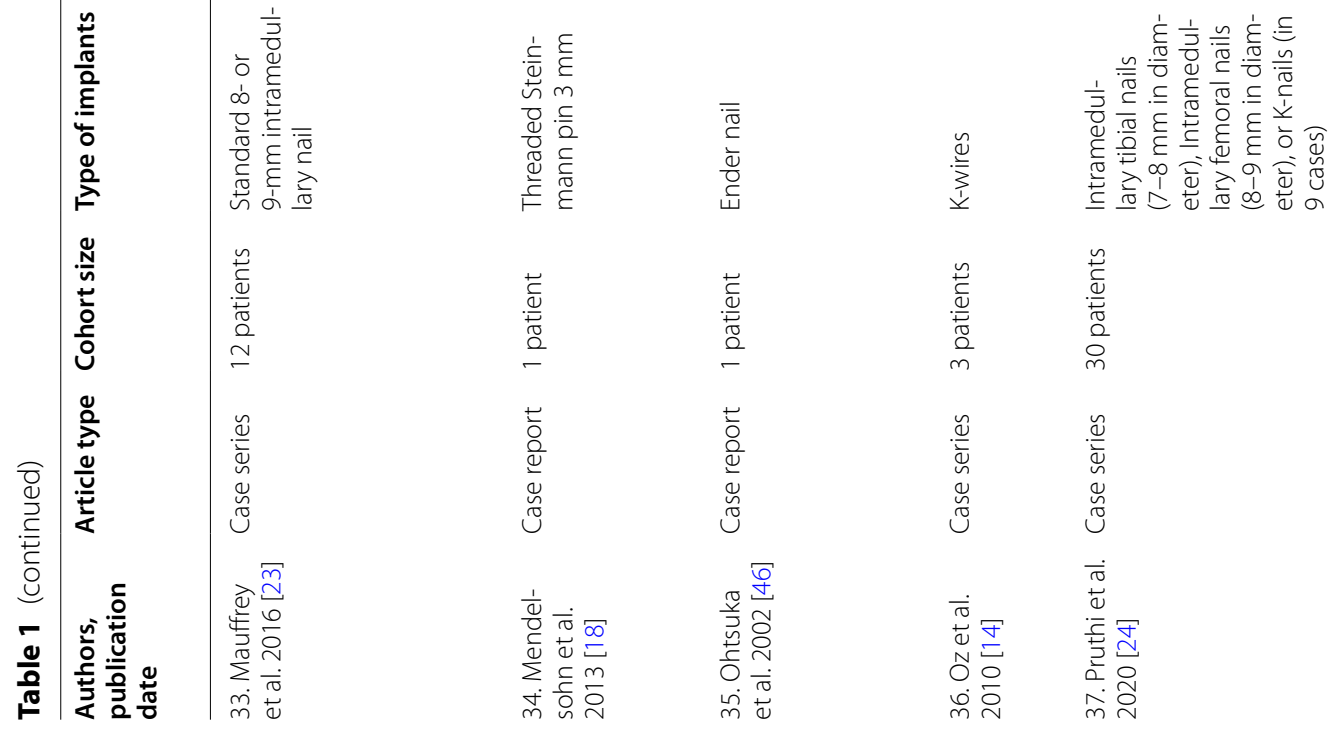

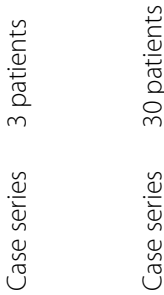

它

率 


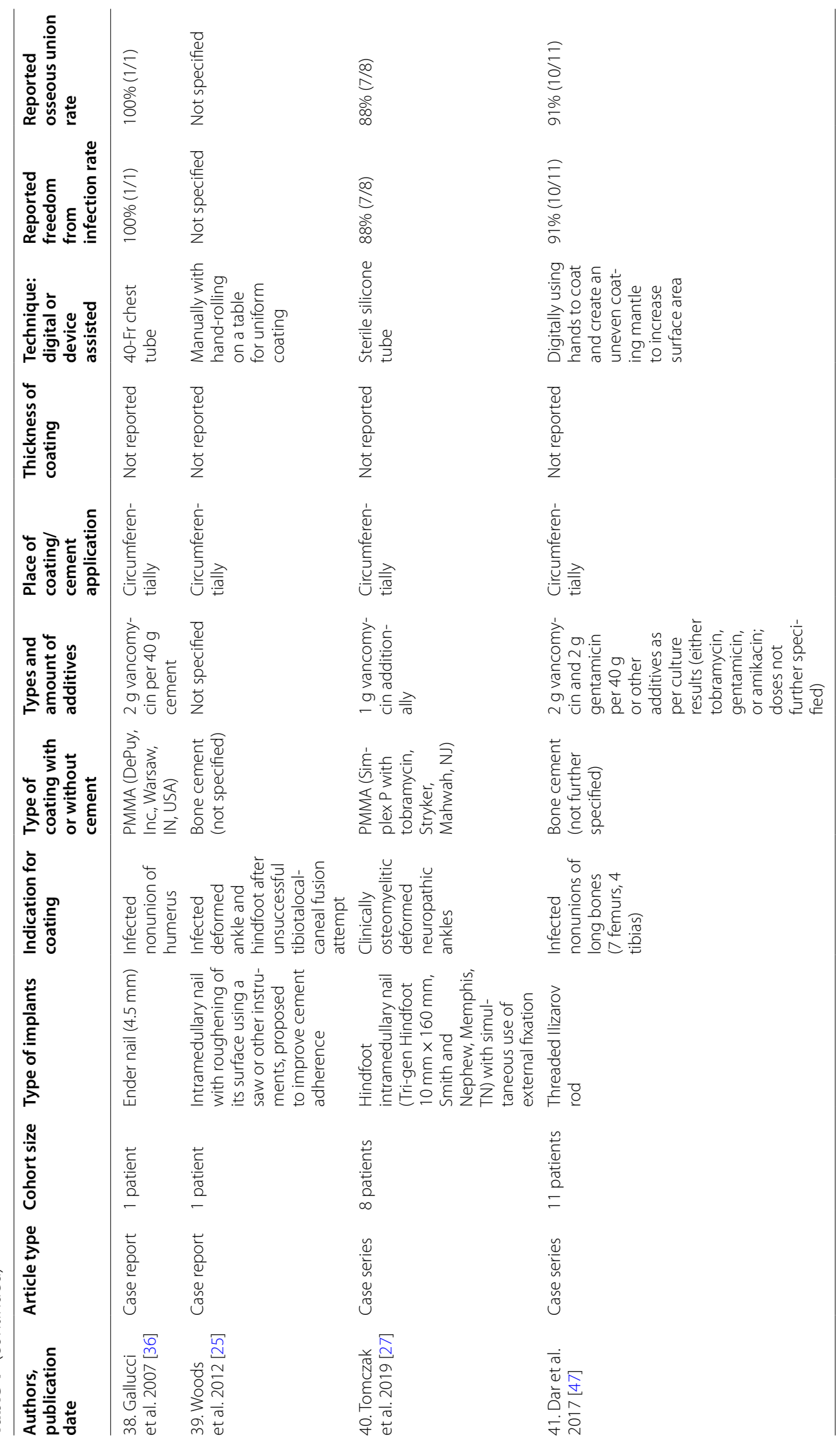


Table 2 Indications for antibiotic-containing cement-coated implants in different studies

\begin{tabular}{|c|c|}
\hline Indications for antibiotic-containing cement-coated implants & Refs. \\
\hline Intramedullary infections of long bones after previous osteosynthesis or nailing & $\begin{array}{l}{[10,11,14,15,} \\
17,21,22,29-32, \\
38,46]\end{array}$ \\
\hline Infected nonunions & $\begin{array}{l}{[8,12,14,16,19} \\
22-24,28,33,36 \\
39-41,44,47,48]\end{array}$ \\
\hline Infected total knee arthroplasty (TKA) & [22] \\
\hline $\begin{array}{l}\text { Segmental bone defects, infected arthrodesis, chronic osteomyelitis with bone defects after debridement, chronic infection after } \\
\text { total knee replacements, infected Charcot ankle, infected bone after distraction osteogenesis }\end{array}$ & [19] \\
\hline Early infection after internal fracture plate fixation with implant retention & [9] \\
\hline Infected open fractures, loosened inverse shoulder prosthesis due to infection & [8] \\
\hline Femoral osteomyelitis with tibial involvement & [42] \\
\hline Chronic osteomyelitis of the tibia and femur & [13] \\
\hline $\begin{array}{l}\text { As part of damage control orthopedics after external fracture fixation in polytrauma patients, to prevent medullary infection during } \\
\text { external fracture fixation }\end{array}$ & [30] \\
\hline Infected fracture internal fixation, treated with coated plates & [44] \\
\hline Osteomyelitis after hindfoot reconstruction for Charcot neuroarthropathy & [26] \\
\hline Secondary osteomyelitis after internal fixation of ankle fracture and subsequent spacer implantation and failure & [45] \\
\hline Infected periprosthetic femoral fracture after open reduction and internal fixation (ORIF) subsequent to total hip arthroplasty & [37] \\
\hline Infected fusion after tibiotalocalcaneal fusion attempt & {$[26,38]$} \\
\hline Infected deformed ankle and hindfoot after unsuccessful tibiotalocalcaneal fusion attempt & [25] \\
\hline Deformed neuropathic ankles with clinical osteomyelitis & [27] \\
\hline Large femoral osteomyelitis defects with a size exceeding $5 \mathrm{~cm}$ after debridement & [34] \\
\hline Infected periprosthetic humeral fracture after total elbow arthroplasty & [35] \\
\hline Tibial nonunions with segmental bone defects ranging from 6 to $25 \mathrm{~cm}$, with an average size of $13 \mathrm{~cm}$ & [23] \\
\hline Comminuted diaphyseal fracture of third metacarpal with bone loss & [18] \\
\hline
\end{tabular}

$(n=1)$. Additionally, one report mentioned the use of PMMA premixed with gentamicin and clindamycin (Copal G+C, Heraeus, Hanau, Germany) with an extra $2 \mathrm{~g}$ colistin added to provide local antibiotic treatment to medullary infection with multiresistant Pseudomonas aeruginosa [28].

\section{Reported molding techniques and instruments}

Instruments used as molds to coat the various implants serving as the metal core of the antibiotic-impregnated cement-coated constructs mainly consisted of chest tubes and silicone tubes of different sizes in accordance to the authors and their desired construct to be implanted (Table 5). Manual or digital application of the coating was used for different implants ranging from rods, plates, nails, and pins. Some authors even used food straws and Teflon tubes $(n=1)$, while others utilized endotracheal tubes $(n=1)$ or metal molds $(n=3)$.

\section{Treatment strategies and outcomes}

Treatment with antibiotic-containing cement- or bone graft substitute-coated orthopedic implants was not only performed as the initial revision procedure to aid in eradication of infection with simultaneous limited construct stability later needing further revision surgery for definitive fixation [10-14, 21, 23, 29-38] $(n=17)$, but also as the main definitive revision procedure with either no further planned procedures $[8,9$, $15,17,19,20,22,24-26,28,39-45](n=18)$ or additional surgery solely to remove the coated implants after completed healing and controlled infection [16, 46 , 47] $(n=3)$. Some studies $(n=2)$ also implemented antibiotic-containing cement-coated implants as part of a three-stage revision procedure protocol $[18,48]$. Others $(n=1)$ used intramedullary coated nails with simultaneous external ring fixation, the latter of which was to be removed after radiological bone healing was seen [27].

In cases where antibiotic-containing cement-coated implants were indicated and used as the definitive single-stage surgical procedure, $10-88 \%$ of them needed additional revision procedures either to control infection or to achieve bony union. The majority $(16 / 18)$ had a required revision rate of $50 \%$ or less. 
Table 3 Various implants used

\begin{tabular}{|c|c|}
\hline Implant used as metal core & Refs. \\
\hline 3-mm beaded intramedullary guidewire & {$[10,29]$} \\
\hline Femoral antegrade and retrograde nails (not further specified) & {$[8,19]$} \\
\hline $\begin{array}{l}\text { Tibial nails (TriGen intramedullary nails, Smith and Nephew, Memphis, Tennessee), knee arthrodesis (not further specified), ankle arthrode- } \\
\text { sis (not further specified) }\end{array}$ & {$[19]$} \\
\hline Tibial nail (UTN, Synthes, Oberdorf, Switzerland) & {$[21]$} \\
\hline 10-mm IM nail (not further specified) & {$[22]$} \\
\hline Intramedullary nail (not further specified) & {$[25]$} \\
\hline Intramedullary tibial nails (7-8 mm in diameter), intramedullary femoral nails (8-9 mm in diameter), or K-nails (in 9 cases) & {$[24]$} \\
\hline Küntscher nails & {$[16,39-41]$} \\
\hline V-nails for tibia (not further specified) & [39] \\
\hline Steinmann pin & {$[17]$} \\
\hline K-wires & {$[11-14]$} \\
\hline $0.062 \mathrm{~K}$-wire or Steinmann pin & [33] \\
\hline Threaded Steinmann pin of $3 \mathrm{~mm}$ diameter & {$[18]$} \\
\hline 3.5-mm Ender nails & {$[21,30,31]$} \\
\hline 4.5-mm Ender nail & {$[36]$} \\
\hline Ender nail (not further specified) & {$[28,46]$} \\
\hline 1.8- or 2-mm llizarov wires & {$[12,15]$} \\
\hline 6-mm llizarov rod & {$[48]$} \\
\hline Ilizarov rod (not further specified) & {$[38,47]$} \\
\hline Two 5-mm llizarov rods connected via hooks and nuts & {$[35]$} \\
\hline Plate osteosynthesis (not further specified) & [9] \\
\hline $\begin{array}{l}\text { Low compression plate (LCP) for tibia, Intramedullary femoral nail (LFN 360/16 mm, Fa Synthes), inverse shoulder prosthesis (Fa Synthes } \\
\text { DePuy) }\end{array}$ & {$[8]$} \\
\hline Steel wires & {$[16]$} \\
\hline Ball-tipped guide wires & {$[15]$} \\
\hline Radiolucent 8.5-mm-diameter carbon-fiber nail (Carbo-Fix, Champlain, IL, USA) & {$[42]$} \\
\hline Radiolucent carbon-fiber intramedullary nail, 10 mm (Carbofix, Orthopedics, Herzliya, Israel) & {$[23]$} \\
\hline Elastic nail (not further specified) & {$[32]$} \\
\hline Internal fixation plates for fibula, radius, and olecranon & {$[44]$} \\
\hline Biomet hindfoot nail for tibiotalocalcaneal fusion & {$[26]$} \\
\hline Expert-HAN (DePuy-Synthes, Spain) tibiotalocalcaneal arthrodesis nail with $10 \mathrm{~mm}$ diameter and $15 \mathrm{~cm}$ length & {$[45]$} \\
\hline 20-hole 4.5-mm narrow limited-contact dynamic compression plate (LC-DCP; Synthes Inc, West Chester, PA) & {$[37]$} \\
\hline 4.5-mm locking compression plate & [34] \\
\hline Hindfoot intramedullary nail (Tri-gen Hindfoot 10 mm × 160 mm, Smith and Nephew, Memphis, TN) & {$[27]$} \\
\hline
\end{tabular}

\section{Complications and their management}

Specific complication rates resulting from antibioticcontaining cement-coated implants ranged from 5\% to $30 \%$ across different studies. This mainly involved nail-cement debonding, nail breakage, nail bending, and migration, occurring in $10-30 \%$ of cases in a series of 20 patients [40]. Further reported complications included joint stiffness, septic arthritis, and more rarely, local antibiotic intolerance due to hypersensitivity [21]. The authors of that report, did not further specify how the hypersensitivity was clinically evident. Nail-cement debonding was more commonly encountered during nail removal and occasionally during nail insertion [20]. Management of this specific complication ranged from use of certain instruments and extraction tools such as J-hooks and additional reaming [43] to creation of a cortical window to aid in cement retrieval [16]. Infection of neighboring joints, suspected to be related to nail insertion site contamination, was managed by using an extracapsular nail insertion point [21]. Nevertheless, the majority of authors did not report any specific complications when using antibiotic-containing cement-coated implants (Table 6). 
Table 4 Summary of type of cement or bone graft substitute and type and quantity of antibiotic additives used for the coating mantle

Bone cement/bone substitute ( \pm premixed antibiotics)
PMMA (DePuy, Inc., Warsaw, IN, USA)
PMMA (Stryker Rutherford)
PMMA, Palacos (Zimmer, Warsaw, Indiana)
Not specified
PMMA cement (Simplex; Howmedica, Rutherford, NJ)
PMMA, Palacos (Heraeus Kulzer, Hanau, Germany)
PMMA, Simplex P bone cement
PMMA, not further specified
PMMA, Palacos
PMMA (Smith and Nephew, TN, USA) (premixed with gentamicin)
Bone cement (not further specified) (premixed with gentamicin)
PMMA (Biomet Cobalt Bone Cement, Warsaw, IN)
Simplex bone cement (Stryker, Mahwah, NJ)
PMMA, Simplex (Antibiotic Simplex, Stryker USA)

PMMA (Palacos R+G, Zimmer, Warsaw, IN, USA) (premixed with $0.5 \mathrm{~g}$ gentamicin)

PMMA cement (not further specified) (premixed with gentamicin)

PMMA bone cement (Simplex, Stryker Orthopedics, Rutherford, NJ)

PMMA bone cement (Simplex, Stryker Orthopedics, Rutherford, NJ)

PMMA, not further specified

\section{PMMA bone cement (PALACOSR)}

PMMA bone cement (Simplex, Stryker, Kalamazoo, MI)

PMMA bone cement (Cobalt, Biomet Orthopedics, Inc., Warsaw, IN)

Hi-Fatigue G Bone Cement (Zimmer) PMMA [premixed with $0.9 \mathrm{~g}$

gentamycin sulfate (0.55 g gentamycin base)]

Simplex bone cement (Stryker, Mahwah, NJ)

PMMA (Stryker, Kalamazoo, MI)

PMMA (not further specified)

PMMA (Copal G+C, Heraeus, Hanau, Germany) (premixed with $1 \mathrm{~g}$ gentamicin and $1 \mathrm{~g}$ clindamycin per $40 \mathrm{~g}$ )

PMMA (not further specified)

Bone cement not specified

PMMA Palacos-R (Zimmer, Warsaw, IN)

PMMA (not further specified)

PMMA (Cemex RX/Tecres Co., Verona, Italy) (premixed with $1.2 \mathrm{~g}$ gentamicin)

PMMA Palacos cement (not further specified)

Bone cement (premixed with gentamicin) (not further specified)

PMMA (DePuy, Inc., Warsaw, IN, USA)

Bone cement (not further specified)

PMMA (Simplex P, premixed with tobramycin, Stryker, Mahwah, NJ)

Bone cement (not further specified)
Antibiotic additives

Refs.

$2.4 \mathrm{~g}$ tobramycin and $2 \mathrm{~g}$ vancomycin per $40 \mathrm{~g}$, vacuum mixing

[10]

$2 \mathrm{~g}$ vancomycin per $40 \mathrm{~g}$

[29]

$3.6 \mathrm{~g}$ tobramycin and $1 \mathrm{~g}$ vancomycin mixed with each 40-g patch of [19] Palacos

$2 \mathrm{~g}$ vancomycin and $2 \mathrm{~g}$ gentamicin mixed per $40 \mathrm{~g}$ bone cement

[39]

$4 \mathrm{~g}$ vancomycin added to each $40 \mathrm{~g}$ PMMA cement

[17]

$2 \mathrm{~g}$ gentamycin per batch of Palacos cement (total two batches used)

$2 \mathrm{~g}$ cefuroxime and $2 \mathrm{~g}$ vancomycin added per $40 \mathrm{~g}$ Simplex P bone cement

$2 \mathrm{~g}$ vancomycin and $2 \mathrm{~g}$ teicoplanin with each $40 \mathrm{~g}$ bone cement

$2 \mathrm{~g}$ vancomycin and $2 \mathrm{~g}$ gentamycin per $40 \mathrm{~g}$ cement

[40]

$2 \mathrm{~g}$ vancomycin with one batch of premixed gentamicin-containing PMMA cement

$4 \mathrm{~g}$ vancomycin mixed with $40 \mathrm{~g}$ gentamicin cement

$3.6 \mathrm{~g}$ tobramycin and $1 \mathrm{~g}$ vancomycin mixed with $40 \mathrm{~g}$ bone cement

1-4 $\mathrm{g}$ antibiotics mixed with $40 \mathrm{~g}$ Simplex for $30 \mathrm{~s}$ before injecting

$4 \mathrm{~g}$ vancomycin and $4 \mathrm{~g}$ tobramycin with $40 \mathrm{~g}$ bone cement

[15]

[48]

$2 \mathrm{~g}$ vancomycin and $2.4 \mathrm{~g}$ tobramycin were each mixed with $40 \mathrm{~g}$ PMMA containing $0.5 \mathrm{~g}$ gentamicin

No additional additives further specified

$2.4 \mathrm{~g}$ tobramycin and $2 \mathrm{~g}$ vancomycin with two packs of $40 \mathrm{~g}$ PMMA

[13]

[30]

$2.4 \mathrm{~g}$ tobramycin and $2 \mathrm{~g}$ vancomycin with two packs of $40 \mathrm{~g}$ PMMA [31]

$4 \mathrm{~g}$ vancomycin (or gentamycin, tobramycin, or imipenem, depending [21] on culture results) per $40 \mathrm{~g}$ PMMA cement

$4 \mathrm{~g}$ vancomycin or $1.6 \mathrm{~g}$ gentamicin per $40 \mathrm{~g}$ PMMA

$2 \mathrm{~g}$ vancomycin and $3.6 \mathrm{~g}$ tobramycin per $40 \mathrm{~g}$ PMMA

$1 \mathrm{~g}$ vancomycin and $3.6 \mathrm{~g}$ tobramycin per $40 \mathrm{~g}$ PMMA

Additional $2.5 \mathrm{~g}$ vancomycin and $1.5 \mathrm{~g}$ tobramycin per $40 \mathrm{~g}$ bone cement

4 vials vancomycin and 4 vials tobramycin

$1 \mathrm{~g}$ vancomycin per $40 \mathrm{~g}$ cement

$3 \mathrm{~g}$ vancomycin and $3.6 \mathrm{~g}$ tobramycin per $40 \mathrm{~g}$ cement

$2 \mathrm{~g}$ colistin per $40 \mathrm{~g}$ cement

$0.5 \mathrm{~g}$ gentamycin and $5 \mathrm{~g}$ vancomycin per $40 \mathrm{~g}$ cement

$3 \mathrm{~g}$ vancomycin and $3.6 \mathrm{~g}$ tobramycin per $40 \mathrm{~g}$ cement [35]

$3 \mathrm{~g}$ vancomycin per $40 \mathrm{~g}$ cement

Additional $3.6 \mathrm{~g}$ tobramycin in presence of polymicrobial Gram-negative cultures

$2 \mathrm{~g}$ vancomycin and $2.4 \mathrm{~g}$ tobramycin per $40 \mathrm{~g}$ cement [18]

No additional antibiotics

$3 \mathrm{~g}$ vancomycin per $40 \mathrm{~g}$ cement

$4 \mathrm{~g}$ vancomycin per $40 \mathrm{~g}$ cement or $2 \mathrm{~g}$ vancomycin and $2.4 \mathrm{~g}$

[24] tobramycin per $40 \mathrm{~g}$ cement

$2 \mathrm{~g}$ vancomycin per $40 \mathrm{~g}$ cement

Not specified

$1 \mathrm{~g}$ vancomycin mixed additionally

$2 \mathrm{~g}$ vancomycin and $2 \mathrm{~g}$ gentamicin per $40 \mathrm{~g}$ cement or other addi-

[47] tives as per culture results (either tobramycin, gentamicin, or amikacin; doses not further specified) 
Table 4 (continued)

\begin{tabular}{|c|c|c|c|}
\hline Bone cement/bone substitute ( \pm premixed antibiotics) & \multicolumn{2}{|c|}{ Antibiotic additives } & Refs. \\
\hline $\begin{array}{l}\text { Cerament G, Cerament V (premixed with either vancomycin or gen- } \\
\text { tamicin) }\end{array}$ & \multicolumn{2}{|c|}{$\begin{array}{l}5 \mathrm{~mL} \text { Cerament } \mathrm{G} \text { ( } 17.5 \mathrm{mg} \text { gentamicin sulfate } / \mathrm{mL} \text { paste), } 10 \mathrm{~mL} \text { Cera- } \\
\text { ment V ( } 66 \mathrm{mg} \text { vancomycin/mL paste) }\end{array}$} & [8] \\
\hline Cerament V (premixed with vancomycin) & \multicolumn{2}{|c|}{10 mL Cerament V (66 mg vancomycin/mL paste) } & {$[26]$} \\
\hline \multicolumn{2}{|c|}{ Application and molding techniques of coating (digital, manual, device assisted) } & \multicolumn{2}{|l|}{ Refs. } \\
\hline Chest tubes & & \multicolumn{2}{|c|}{$\begin{array}{l}{[10,11,13-16,18,21,23,24,29-31} \\
35,36,42,48]\end{array}$} \\
\hline Silicone tubes & & \multicolumn{2}{|c|}{$[12,19,20,22,27,28,32,33,43-45]$} \\
\hline Manually with digital hand-rolling & & \multicolumn{2}{|l|}{$[25,39,41]$} \\
\hline Manual application without mention of hand-rolling & & \multicolumn{2}{|l|}{$[9,17,34,47]$} \\
\hline Manual application using a syringe & & \multicolumn{2}{|l|}{$[8,26]$} \\
\hline Steel/metal molds & & \multicolumn{2}{|l|}{$[20,24,43]$} \\
\hline Food straws, Teflon tubes & & \multicolumn{2}{|l|}{ [12] } \\
\hline Endotracheal tubes & & \multicolumn{2}{|l|}{$[40]$} \\
\hline Not specified & & \multicolumn{2}{|l|}{$[37,38,46]$} \\
\hline
\end{tabular}

Table 6 Encountered complications

\begin{tabular}{ll}
\hline Encountered complications reported & Refs. \\
\hline Broken antibiotic cement nail/rod & {$[10,14,20,29,40,41]$} \\
Cement-nail debonding & {$[19,20,43]$} \\
Cement cracking & {$[10]$} \\
Nail migration & {$[40]$} \\
Distal locking screw migration & {$[22]$} \\
Nail bending & {$[40]$} \\
Difficult nail removal & {$[16,19,20,29,40,41]$} \\
Adjacent knee-joint infection & {$[29]$} \\
Septic hip arthritis likely from insertion site contamination & {$[21]$} \\
Limited range of knee-joint motion & {$[39]$} \\
Knee stiffness & {$[41]$} \\
Union failure & {$[16,22,24,47]$} \\
Persistent and/or recurring infection & {$[22,24,34]$} \\
Amputation & {$[22,27,29,43]$} \\
Nerve compression, painful screw, hematoma, skin infection, and joint contractures & {$[20]$} \\
Painful olecranon plate needing removal & {$[44]$} \\
Local antibiotic intolerance related to vancomycin hypersensitivity & {$[21]$} \\
Pin-site infection, wound dehiscence, proximal tibial fracture & {$[27]$} \\
No specific complications reported & {$[8,9,11-13,15,17,18,23,25$,} \\
& $26,28,30-33,35-38,42,45$, \\
\hline
\end{tabular}

\section{Discussion}

After a thorough appraisal of the available literature reporting specifically on antibiotic-containing cement and bone graft substitute coating techniques for orthopedic implants in an operative clinical setting,
41 articles with a collective total number of 607 cases were identified. Available reviews related to this topic are scarce and have so far focused on general indications, efficacy, and outcomes with no specific detailed description of the different coating techniques and 
their associated particularities in an intraoperative setting [49-51].

To date, there are no general consensus and specific guidelines on the particular techniques used to cementcoat implants in a custom-made fashion. The available evidence is limited to case reports and case series from different groups reporting on their custom-made coating techniques as part of different treatment protocols in various clinical settings. Some authors used this treatment method not only as part of a staged treatment protocol with the initial aim of infection control followed by definitive fixation for bone healing but also as the sole surgical procedure to treat infected nonunions and posttraumatic osteomyelitis [17, 19, 39-41].

Management of infected nonunions and posttraumatic osteomyelitis is multifactorial and involves several components mainly consisting of removal of infected hardware, adequate thorough debridement of infected bone and tissues, appropriate dead space management with local antibiotic delivery to control infection, and, if necessary, adequate soft tissue coverage and bone defect reconstruction $[10,52]$. Use of local antibiotic delivery methods, with reported results comparable to treatment with systemic antibiotics alone [53, 54], can help reduce the burden of toxicity associated with systemic antibiotics and address poor penetration from poor vasculature and biofilm formation at the site of infections as well as potential development of antibiotic resistance [40].

Aside from high concentrations of antibiotics needed to control infection, bone stability must also be provided for adequate bone healing and union to occur, particularly in cases of infected fractures or nonunions. This generally entails a staged treatment protocol with usually two planned procedures being necessary with provisional stability being provided through means of external fixation, casts, or splints [12, 40]. However, complications such as pin-site infections, joint stiffness, contractures, and others related to patient compliance limit the usefulness of external fixation [55].

To reduce the burden of treatment and improve outcomes, antibiotic-impregnated cement-coated nails, offering both local antibiotic delivery and adequate internal bone stability allowing for simultaneous control of infection and osseous union, were introduced around 20 years ago [10]. However, and as evident in this review, there is no general uniform method of applying this form of surgical treatment. Accordingly, an individualized treatment strategy can be tailored as necessary.

To provide the required stability for osseous union across the site of infected bone, various implants were used as the metal core of the antibiotic-containing cement-coated construct. Because guidewires, K-wires, Ilizarov wires, and nails such as Ender or Küntscher nails do not necessarily offer the stability required for bone healing, intramedullary nails have been coated and implanted as means of definitive internal fixation [19]. This not only avoids the need for external stabilizing systems but can also, more importantly, allow for weightbearing, which in turn improves outcomes and reduces complications [19]. From an antibiotic elution properties perspective, coated interlocking nails have been shown to have better antibiotic delivery characteristics in comparison with coated guidewires, potentially from a thinner cement mantle and cooler associated exothermic reactions [56], further supporting their use particularly when a definitive one-stage procedure with the needed adequate mechanical stability is indicated. Choice of the particular implant to be coated should be made on an individual, case-dependent basis considering both patient characteristics and anatomical particularities. In addition to intramedullary nails, arthrodesis nails, plates, and joint replacement prosthesis have also been coated with antibiotic-containing cement, with favorable outcomes. More recently, carbon-fiber nails [42] have been applied as they are radiolucent and reduce the production of artifacts, particularly on radiological follow-up with magnetic resonance imaging (MRI), thus allowing for more accurate treatment monitoring. However, radiological follow-up by MRI is not standard, and its clinical use after surgical interventions remains questionable. Interestingly, with regards to bacterial adhesion on implant surfaces, carbon-fiber rods did not demonstrate inferior results compared with steel rods in an experimental study potentially supporting their use as a sound alternative option to conventional metallic implants [57].

The most specific reported complication potentially arising after use of cement-coated implants is nailcement debonding. In the largest series, involving more than 100 patients, Conway et al. [20] reported encountering this complication in 23 from a total of 110 cases $(\sim 21 \%)$ during both insertion and removal of the antibiotic-containing cement-coated implants. Other authors encountered this complication in approximately $10-30 \%$ of cases [10, 19, 29, 40, 41, 43]. Management mainly involved removal of retained debonded cement with use of certain extraction tools, such as J-hooks from hip arthroplasty instrumentation set [43], and additional reaming. In some cases, creation of a cortical window to facilitate retrieval may be necessary [16]. Moreover, use of a threaded metal core has been suggested to prevent occurrence of cement debonding upon removal [49], and in one report the authors proposed roughening the surface of the IM nail using saws and other instruments before coating to improve cement adherence [25].

Different antibiotic agents have been used as additives to mix with bone cement in accordance with 
susceptibility testing [6]. Vancomycin was the most commonly used antibiotic in all studies; this corresponds with methicillin-resistant Staphylococcus aureus (MRSA) being the most frequent causative pathogen [20]. Aminoglycosides, such as gentamicin, have also been shown to be heat-stable with low allergic potential, making them suitable as antibiotic additives [58]. Different bone cement formulations have been associated with various elution properties when certain antibiotics are added to them, with Palacos bone cement having generally more favorable properties in comparison with Simplex bone cement [59-61]. Furthermore, use of a combination of different antibiotic agents, additionally mixed, was shown to improve elution properties [62, 63]. PMMA bone cement was most commonly used to prepare the coated implant. Moreover, custom-made intraoperative addition of antibiotics to the cement formulation has been shown to provide better elution properties in comparison with commercially premixed antibiotic-containing cement preparations [64, 65], further supporting custom-made intraoperative preparation of these constructs in accordance with antimicrobial susceptibility testing. More recently, bone graft substitutes, composed of calcium phosphate and calcium hydroxyapatite, premixed with either vancomycin or gentamicin have been utilized to coat orthopedic implants $[8,26]$. Comprehensive evidence elaborating on the microbiological and biomechanical properties of such bone graft substitutes in the coating of orthopedic implants, to treat infected fractures and nonunions, in comparison with the conventional method using bone cement is still very limited, and lately encouraging results have been reported when CERAMENT G was applied as part of a one-stage treatment protocol of chronic osteomyelitis [66].

Molding techniques varied across different studies. A uniform circumferentially applied antibiotic-containing cement mantle was created either manually, through rolling, or with the aid of certain molds. Thonse et al. [19] introduced the silicone tubing technique and reported better and more time-efficient coating with the use of this method as opposed to the previously used stainless-steel molds. Most other groups used chest tubes as a molding instrument. Retrieval of the coated nail was usually performed after the cement was allowed to set [19]. Some authors submerged the construct in a bowl containing cool sterile water to prevent heat accumulation and potential plastic tube melting during the exothermic phase [67]. To avoid breakage of the cement mantle during retrieval of the coated nail before insertion, sterile mineral oil was used to lubricate the inner mold surface, allowing for faster fabrication time and easier tube removal [68]. With regards to the antibiotic elution properties, one study showed no difference in the elution properties of tobramycin with or without use of mineral oil [69].

In cases where bone defects are present after thorough debridement, treatment with antibiotic-containing cement-coated nails has been associated with varying results. To assess the efficacy of cement-coated implants within the scope of revision surgery to surgically treat infected nonunions with bone defects and remaining in situ fixators, Shyam et al. [39] conducted a study involving 25 patients. The reported outcomes from this study demonstrated more unfavorable results with increasing size of the bone defect left after debridement of infected nonunion to be treated and suggested the use of alternative treatment methods when defects exceeded $6 \mathrm{~cm}$ in size. In this report, patients with large defects required additional surgery in the form of debridement and application of an Ilizarov ring fixator. Four out of five patients achieved union. One patient developed a stiff nonunion and was mobilized with a brace after declining further surgery [39]. On the contrary, Mauffrey et al. [23] reported good outcomes with use of antibiotic-containing cement-coated intramedullary nails in the two-stage treatment of 12 patients with infected tibial nonunions and segmental bone defects ranging from 6 to $25 \mathrm{~cm}$ in size. Moreover, Yu et al. [34] demonstrated a $100 \%$ union rate and a $92 \%$ freedom from infection rate in a sample of 13 patients with chronic femoral osteomyelitis and remaining defects exceeding $5 \mathrm{~cm}$ in size after debridement, and a mean defect size of more than $9 \mathrm{~cm}$, when using antibiotic-containing cement-coated plates for the first stage as part of a two-stage induced membrane treatment protocol. Thus, more elaborate evidence is needed to conclude as to which antibioticcontaining cement-coated construct, consisting of either intramedullary nails or plates, is more effective in the two-stage treatment of large bone defects.

Overall, high rates of infection control and bony union are possible with the use of antibiotic-containing cement-coated implants in particular clinical settings. Outcomes and major complications, especially when used in form of a one-stage definitive procedure, have been comparable between the reporting studies with variable patient collectives. Higher rates of infection control were achievable through use of antibioticcontaining cement-coated implants than osseous union rates. Bony union rates increased and ranged from $70 \%$ to $100 \%$ of cases after additional procedures involving exchange nailing and bone grafting were performed. Despite the mentioned complications, the results presented in this review can be regarded as advantageous when using this form of surgical treatment when indicated. 


\section{Conclusions}

Cement coating of orthopedic implants is supportive and sometimes necessary in various clinical settings. Adequate cement coating techniques can reduce the burden of treatment and be associated with favorable outcomes, particularly in revision surgery. Downsides observed with current cement coating techniques are debonding of cement during implant removal and breakage of the coated metallic implant. After reviewing the available evidence reporting on different custom-made cement coating techniques with their respective benefits and limitations applied so far, it is evident that the perfect all-in-one implant cement coating method has yet to be found, and that a reasonable amount of heterogeneity is present in reported literature. Further prospective targeted research on these cement coating methods in different operative settings is warranted to better optimize patient care and outcomes when applying these techniques.

\section{Acknowledgements}

None.

\section{Authors' contributions}

A.I. collected and analyzed data, drafted, reviewed, and critically revised the manuscript. N.W., S.B., J.M., S.L., and M.K. analyzed data and reviewed the manuscript. V.A. provided administrative and supervisory support, reviewed, and critically revised the manuscript. M.R. conceptualized the manuscript, provided technical and supervisory support, reviewed and critically revised the manuscript. All authors read and approved the final manuscript.

\section{Funding}

This research did not receive any specific grant from funding agencies in the public, commercial, or not-for-profit sectors.

\section{Availability of data and materials}

The datasets used and/or analyzed during the current study are available from the corresponding author on reasonable request.

\section{Declarations}

Ethics approval and consent to participate

Not applicable.

\section{Consent for publication}

Not applicable.

\section{Competing interests}

The authors declare that they have no conflict of interest.

\section{Author details}

${ }^{1}$ Department of Trauma Surgery, University Medical Center Regensburg, Franz-Josef-Strauß-Allee 11, 93053 Regensburg, Germany. ${ }^{2}$ Experimental Rheumatology Unit, Department of Orthopedics, Jena University Hospital, Waldkliniken Eisenberg GmbH, Klosterlausnitzer Strasse 81, 07607 Eisenberg, Germany.

Received: 11 March 2021 Accepted: 17 November 2021

Published online: 23 December 2021

\section{References}

1. Charnley J (1960) Anchorage of the femoral head prosthesis to the shaft of the femur. J Bone Jt Surg Br. 42-b:28-30
2. Parvizi J, Saleh KJ, Ragland PS, Pour AE, Mont MA (2008) Efficacy of antibiotic-impregnated cement in total hip replacement. Acta Orthop 79(3):335-341

3. Buchholz HW, Engelbrecht H (1970) Depot effects of various antibiotics mixed with Palacos resins. Chirurg 41(11):511-515

4. Klemm K (1979) Gentamicin-PMMA-beads in treating bone and soft tissue infections (author's transl). Zentralbl Chir 104(14):934-942

5. Kelm J, Bohrer P, Schmitt E, Anagnostakos K (2009) Treatment of proximal femur infections with antibiotic-loaded cement spacers. Int J Med Sci 6(5):258-264

6. Bistolfi A, Massazza G, Verné E et al (2011) Antibiotic-loaded cement in orthopedic surgery: a review. ISRN Orthop 2011:290851

7. Raschke MJ, Schmidmaier G (2004) Biologisierung von Implantaten in der Chirurgie des Stütz- und Bewegungsapparates. Unfallchirurg 107(8):653-663

8. Freischmidt H, Armbruster J, Reiter G, Grützner PA, Helbig L, Guehring T (2020) Individualized techniques of implant coating with an antibioticloaded, hydroxyapatite/calcium sulphate bone graft substitute. Ther Clin Risk Manag 16:689-694

9. Qiu X-S, Cheng B, Chen Y-X, Qi X-Y, Sha W-P, Chen G-Z (2018) Coating the plate with antibiotic cement to treat early infection after fracture fixation with retention of the implants: a technical note. BMC Musculoskelet Disord 19(1):360

10. Paley D, Herzenberg JE (2002) Intramedullary infections treated with antibiotic cement rods: preliminary results in nine cases. J Orthop Trauma 16(10):723-729

11. Wasko MK, Borens O (2013) Antibiotic cement nail for the treatment of posttraumatic intramedullary infections of the tibia: midterm results in 10 cases. Injury 44(8):1057-1060

12. Bharti A, Saroj UK, Kumar V, Kumar S, Omar BJ (2016) A simple method for fashioning an antibiotic impregnated cemented rod for intramedullary placement in infected non-union of long bones. J Clin Orthop Trauma 7(Suppl 2):171-176

13. Bar-On E, Weigl DM, Bor N et al (2010) Chronic osteomyelitis in children: treatment by intramedullary reaming and antibiotic-impregnated cement rods. J Pediatr Orthop 30(5):508-513

14. Oz M, Walter G, Walter G (2010) Temporary intramedullary stabilisation of infected non-union in long bones with local antibiotic-impregnated cement rods: case reports. Z Orthop Unfall 148(5):559-565

15. Reilly RM, Robertson T, O'Toole RV, Manson TT (2016) Are antibiotic nails effective in the treatment of infected tibial fractures? Injury 47(12):2809-2815

16. Selhi HS, Mahindra P, Yamin M, Jain D, De Long WG Jr, Singh J (2012) Outcome in patients with an infected nonunion of the long bones treated with a reinforced antibiotic bone cement rod. J Orthop Trauma 26(3):184-188

17. Fan C-Y, Hsieh M-S, Chen W-M, Chen C-F (2011) Successful management of infected intramedullary nailing with reaming, lavage, and insertion of antibiotic-impregnated cement rods. J Exp Clin Med 3(3):137-141

18. Mendelsohn ES, Warganich T, Nielsen ES, Najibi S (2013) Application of an antibiotic intramedullary nail in the management of a large metacarpal bone defect. Tech Hand Up Extrem Surg 17(4):187-191

19. Thonse R, Conway JD (2008) Antibiotic cement-coated nails for the treatment of infected nonunions and segmental bone defects. J Bone Jt Surg Am 90(Suppl 4):163-174

20. Conway J, Mansour J, Kotze K, Specht S, Shabtai L (2014) Antibiotic cement-coated rods: an effective treatment for infected long bones and prosthetic joint nonunions. Bone Jt J 96-b(10):1349-1354

21. Sancineto CF, Barla JD (2008) Treatment of long bone osteomyelitis with a mechanically stable intramedullar antibiotic dispenser: nineteen consecutive cases with a minimum of 12 months follow-up. J Trauma 65(6):1416-1420

22. Makhdom AM, Buksbaum J, Rozbruch SR, Da Cunha R, Fragomen AT (2020) Antibiotic cement-coated interlocking intramedullary nails in the treatment of septic complex lower extremity reconstruction; a retrospective analysis with two year minimum follow up. J Bone Jt Infect 5(4):176-183

23. Mauffrey C, Hake ME, Chadayammuri V, Masquelet AC (2016) Reconstruction of long bone infections using the induced membrane technique: tips and tricks. J Orthop Trauma 30(6):e188-193 
24. Pruthi V, Ummat A, Singh M et al (2020) Use of custom-made antibiotic coated intra-medullary nail in treatment of infected non-union of long bones. Indian J Public Health Res Dev 11(2):802-807

25. Woods JB, Lowery NJ, Burns PR (2012) Permanent antibiotic impregnated intramedullary nail in diabetic limb salvage: a case report and literature review. Diabet Foot Ankle 3:11908

26. Anugraha A, Hughes LD, Pillai A (2019) A novel technique for fabricating antibiotic-coated intramedullary nails using an antibiotic-loaded calcium sulphate hydroxyapatite bio-composite, Cerament-V. J Surg Case Rep 2019(11):rjz327

27. Tomczak C, Beaman D, Perkins S (2019) Combined intramedullary nail coated with antibiotic-containing cement and ring fixation for limb salvage in the severely deformed, infected, neuroarthropathic ankle. Foot Ankle Int 40(1):48-55

28. Senn D, Gehmert S, Ochsner PE, Kühn K-D, Nowakowski AM (2017) Therapy for chronic recurrent osteomyelitis with multi-resistant Pseudomonas aeruginosa using local antibiotic release by a polymethylmethacrylate custom-made tibia nail. Surg Infect Case Rep 2(1):26-30

29. Qiang Z, Jun PZ, Jie XJ, Hang L, Bing LJ, Cai LF (2007) Use of antibiotic cement rod to treat intramedullary infection after nailing: preliminary study in 19 patients. Arch Orthop Trauma Surg 127(10):945-951

30. Bhadra AK, Roberts CS (2009) Indications for antibiotic cement nails. J Orthop Trauma 23(5 Suppl):S26-30

31. Madanagopal SG, Seligson D, Roberts CS (2004) The antibiotic cement nail for infection after tibial nailing. Orthopedics 27(7):709-712

32. Bu ZY, Hu LJ, Li C, Li AJ (2020) Clinical analysis of application of antibiotic bone cement spacer combined with membrane induction technology in treatment of osteomyelitis after femoral intramedullary nail operation-a case series. JPMA 70(2):360-362

33. Mendicino RW, Bowers CA, Catanzariti AR (2009) Antibiotic-coated intramedullary rod. J Foot Ankle Surg 48(2):104-110

34. Yu X, Wu H, Li J, Xie Z (2017) Antibiotic cement-coated locking plate as a temporary internal fixator for femoral osteomyelitis defects. Int Orthop 41(9):1851-1857

35. Liporace FA, Kaplan D, Stickney W, Yoon RS (2014) Use of a hinged antibiotic-loaded cement spacer for an infected periprosthetic fracture in a total elbow arthroplasty: a novel construct utilizing Ilizarov rods: a case report. JBJS Case Connect 4(4):e122

36. Gallucci G, Donndorff A, Boretto J, Constantini J, De Carli P (2007) Infected nonunion of the humerus treated with an antibiotic cement rod. Case report. Chir Main 26(4-5):242-246

37. Liporace FA, Yoon RS, Frank MA et al (2012) Use of an "antibiotic plate" for infected periprosthetic fracture in total hip arthroplasty. J Orthop Trauma 26(3):e18-23

38. Miller J, Hoang V, Yoon RS, Liporace FA (2017) Staged treatment of infected tibiotalar fusion using a combination antibiotic spacer and antibiotic-coated intramedullary nail. J Foot Ankle Surg 56(5):1099-1103

39. Shyam AK, Sancheti PK, Patel SK, Rocha S, Pradhan C, Patil A (2009) Use of antibiotic cement-impregnated intramedullary nail in treatment of infected non-union of long bones. Indian J Orthop 43(4):396-402

40. Bhatia C, Tiwari AK, Sharma SB, Thalanki S, Rai A (2017) Role of antibiotic cement coated nailing in infected nonunion of tibia. Malays Orthop J 11(1):6-11

41. Pradhan C, Patil A, Puram C, Attarde D, Sancheti P, Shyam A (2017) Can antibiotic impregnated cement nail achieve both infection control and bony union in infected diaphyseal femoral non-unions? Injury 48:S66-S71

42. Mauffrey C, Chaus GW, Butler N, Young H (2014) MR-compatible antibiotic interlocked nail fabrication for the management of long bone infections: first case report of a new technique. Patient Saf Surg 8(1):14

43. Thonse R, Conway J (2007) Antibiotic cement-coated interlocking nail for the treatment of infected nonunions and segmental bone defects. J Orthop Trauma 21(4):258-268

44. Conway JD, Hlad LM, Bark SE (2015) Antibiotic cement-coated plates for management of infected fractures. Am J Orthop 44(2):E49-E53

45. Herrera-Pérez M, Boluda-Mengod J, Gutierrez-Morales MJ, Pais-Brito ال (2017) Tibiotalocalcaneal fusion with a cemented coated retrograde nail as a salvage procedure for infected ORIF of the ankle. Rev Esp Cir Ortop Traumatol 61(6):441-445
46. Ohtsuka H, Yokoyama K, Higashi K et al (2002) Use of antibioticimpregnated bone cement nail to treat septic nonunion after open tibial fracture. J Trauma 52(2):364-366

47. Dar TA, Dhar SA, Mir NA, Maajid S, Dar RA, Hussain A (2017) Antibiotic impregnated cement coated ilizarov rod for the management of infected non union of long bone. Acta Orthop Belg 83(4):521-526

48. Cho JW, Kim J, Cho WT, Kent WT, Kim HJ, Oh JK (2018) Antibiotic coated hinged threaded rods in the treatment of infected nonunions and intramedullary long bone infections. Injury 49(10):1912-1921

49. Koury KL, Hwang JS, Sirkin M (2017) The antibiotic nail in the treatment of long bone infection: technique and results. Orthop Clin North Am 48(2):155-165

50. Wasko MK, Kaminski R (2015) Custom-made antibiotic cement nails in orthopaedic trauma: review of outcomes, new approaches, and perspectives. Biomed Res Int 2015:387186

51. Nizegorodcew T, Palmieri G, Marzetti E (2011) Antibiotic-coated nails in orthopedic and trauma surgery: state of the art. Int J Immunopathol Pharmacol 24(1 Suppl 2):125-128

52. Patzakis MJ, Zalavras CG (2005) Chronic posttraumatic osteomyelitis and infected nonunion of the tibia: current management concepts. J Am Acad Orthop Surg 13(6):417-427

53. Patzakis MJ, Mazur K, Wilkins J, Sherman R, Holtom P (1993) Septopal beads and autogenous bone grafting for bone defects in patients with chronic osteomyelitis. Clin Orthop Relat Res 295:112-118

54. Calhoun JH, Henry SL, Anger DM, Cobos JA, Mader JT (1993) The treatment of infected nonunions with gentamicin-polymethylmethacrylate antibiotic beads. Clin Orthop Relat Res 295:23-27

55. Green SA (1983) Complications of external skeletal fixation. Clin Orthop Relat Res 180:109-116

56. Karek MR, Jackson NM, Flynn JC, Vaidya R, Markel DC (2017) Elution profiles of two methods of antibiotic tibial nail preparations. Orthopedics 40(3):e436-e442

57. Frieler S, Geßmann J, Jettkant B et al (2019) Temporary arthrodesis of the knee in two-stage septic prosthesis exchange: in vitro analysis of adhesion of Staphylococcus aureus on steel and carbon fiber rods. Orthopade 48(2):130-135

58. Zalavras CG, Patzakis MJ, Holtom P (2004) Local antibiotic therapy in the treatment of open fractures and osteomyelitis. Clin Orthop Relat Res 427:86-93

59. Meeker DG, Cooper KB, Renard RL, Mears SC, Smeltzer MS, Barnes CL (2019) Comparative study of antibiotic elution profiles from alternative formulations of polymethylmethacrylate bone cement. J Arthroplasty 34(7):1458-1461

60. Lee SH, Tai CL, Chen SY, Chang CH, Chang YH, Hsieh PH (2016) Elution and mechanical strength of vancomycin-loaded bone cement: in vitro study of the influence of brand combination. PLOS ONE 11(11):e0166545

61. Stevens CM, Tetsworth KD, Calhoun JH, Mader JT (2005) An articulated antibiotic spacer used for infected total knee arthroplasty: a comparative in vitro elution study of Simplex and Palacos bone cements. J Orthop Res 23(1):27-33

62. Penner MJ, Masri BA, Duncan CP (1996) Elution characteristics of vancomycin and tobramycin combined in acrylic bone-cement. J Arthroplasty 11(8):939-944

63. Slane J, Gietman B, Squire M (2018) Antibiotic elution from acrylic bone cement loaded with high doses of tobramycin and vancomycin. J Orthop Res 36(4):1078-1085

64. Frew NM, Cannon T, Nichol T, Smith TJ, Stockley I (2017) Comparison of the elution properties of commercially available gentamicin and bone cement containing vancomycin with 'home-made' preparations. Bone Jt J 99-b(1):73-77

65. Boelch SP, Jordan MC, Arnholdt J, Rudert M, Luedemann M, Steinert AF (2017) Loading with vancomycin does not decrease gentamicin elution in gentamicin premixed bone cement. J Mater Sci Mater Med 28(7):104

66. McNally MA, Ferguson JY, Lau AC et al (2016) Single-stage treatment of chronic osteomyelitis with a new absorbable, gentamicin-loaded, calcium sulphate/hydroxyapatite biocomposite: a prospective series of 100 cases. Bone Jt J 98-b(9):1289-1296 
67. Mauffrey C, Butler N, Hake ME (2016) Fabrication of an interlocked antibiotic/cement-coated carbon fiber nail for the treatment of long bone osteomyelitis. J Orthop Trauma 30(Suppl 2):S23-S24

68. Kim JW, Cuellar DO, Hao J, Seligson D, Mauffrey C (2014) Custom-made antibiotic cement nails: a comparative study of different fabrication techniques. Injury 45(8):1179-1184

69. Lindner JR, Seligson D, Chisena E, Jortani SA, Korte EA (2020) Does an antibiotic nail made in a lubricated tube release antibiotic? Eur J Orthop Surg Traumatol 30(1):153-156

\section{Publisher's Note}

Springer Nature remains neutral with regard to jurisdictional claims in published maps and institutional affiliations.

\section{Submit your manuscript to a SpringerOpen ${ }^{\circ}$ journal and benefit from:}

- Convenient online submission

- Rigorous peer review

- Open access: articles freely available online

- High visibility within the field

- Retaining the copyright to your article

Submit your next manuscript at $\mathbf{s p r i n g e r o p e n . c o m ~}$ 